NBER WORKING PAPER SERIES

\title{
RISK PRICE DYNAMICS
}

Jaroslav Borovička

Lars Peter Hansen

Mark Hendricks

José A. Scheinkman

WORKING PAPER 15506

http://www.nber.org/papers/w15506

\section{NATIONAL BUREAU OF ECONOMIC RESEARCH \\ 1050 Massachusetts Avenue \\ Cambridge, MA 02138 \\ November 2009}

This paper was originally presented as the Journal of Financial Econometrics Lecture at the June 2008 SoFiE conference. We gratefully acknowledge support by the National Science Foundation under Award Numbers SES0519372 (Borovička, Hansen and Hendricks) and SES0718407 (Scheinkman) The views expressed herein are those of the author(s) and do not necessarily reflect the views of the National Bureau of Economic Research.

(C) 2009 by Jaroslav Borovička, Lars Peter Hansen, Mark Hendricks, and José A. Scheinkman. All rights reserved. Short sections of text, not to exceed two paragraphs, may be quoted without explicit permission provided that full credit, including $(\subset)$ notice, is given to the source. 
Risk Price Dynamics

Jaroslav Borovička, Lars Peter Hansen, Mark Hendricks, and José A. Scheinkman

NBER Working Paper No. 15506

November 2009

JEL No. C52,E44,G12

\section{ABSTRACT}

We present a novel approach to depicting asset pricing dynamics by characterizing shock exposures and prices for alternative investment horizons. We quantify the shock exposures in terms of elasticities that measure the impact of a current shock on future cash-flow growth. The elasticities are designed to accommodate nonlinearities in the stochastic evolution modeled as a Markov process. Stochastic growth in the underlying macroeconomy and stochastic discounting in the representation of asset values are central ingredients in our investigation. We provide elasticity calculations in a series of examples featuring consumption externalities, recursive utility, and jump risk.

Jaroslav Borovička

Department of Economics

The University of Chicago

1126 East 59th Street

Chicago, IL 60637

borovicka@uchicago.edu

Lars Peter Hansen

Department of Economics

The University of Chicago

1126 East 59th Street

Chicago, IL 60637

and NBER

l-hansen@uchicago.edu

\author{
Mark Hendricks \\ Department of Economics \\ The University of Chicago \\ 1126 East 59th Street \\ Chicago, IL 60637 \\ hendricks@uchicago.edu \\ José A. Scheinkman \\ Department of Economics \\ Princeton University \\ Princeton, NJ 08544-1021 \\ and NBER \\ joses@princeton.edu
}




\section{Introduction}

We propose a new way to characterize risk price dynamics. In the methods of mathematical finance, risk prices are encoded using the familiar risk neutral transformation and the instantaneous risk-free rate. In structural models of macroeconomic risk, they are encoded in the stochastic discount factor process used to represent prices at alternative payoff horizons. As an alternative, we depict asset pricing dynamics by extending two types of methods: local risk prices and impulse response functions. Local risk prices give the reward expressed in terms of expected returns for alternative local exposures to risk such as shocks to the macro-economy. Impulse response functions characterize how shocks today contribute to future values of a stochastic process such as macroeconomic growth or future cash flows. First we develop a related concept but tailored to the pricing of the exposure to macroeconomic risk, and then we extend the concept of a local risk price by asking how the reward to shock exposure changes as we alter the terminal or maturity date for the payoff. This leads us to construct shock-exposure and shock-price elasticities as functions of payoff horizons. Structural asset pricing models feature state dependence in risk premia as well as sensitivity to the payoff horizon. These risk premia depend on shock exposures and prices, and the elasticities we propose reflect both dependencies.

We believe that uncertainty about macroeconomic growth has important welfare implications and major consequences to market valuations of forward-looking assets. To explore these phenomena requires the simultaneous study of stochastic growth and discounting, in contrast to the extensive literature on fixed income securities and the term structure of interest rates that abstracts from growth. Previous work has sought to provide informative characterizations of risk premia for cash flows that grow stochastically over time and to extract the distinct contributions of risk exposure (the asset pricing counterpart to a quantity) and risk prices. See, for instance, Lettau and Wachter (2007), Hansen and Scheinkman (2009a,b) and Hansen (2009).

While there have been quantitative and empirical successes through the use of ad hoc models of stochastic discount factors specified flexibly to enforce the absence of arbitrage, we continue to be interested in structural models that allow us to truly answer the question "how does risk or uncertainty get priced?" The promise of such models is that they will allow researchers to assign values to the shocks identified in macroeconomic models and support welfare analyses that are linked to uncertainty. While reduced-form models continue to provide a convenient shortcut for presenting empirical evidence, we aim to provide a dynamic characterization of risk pricing that will support structural investigations that stretch models beyond the support of the existing data.

The methods we develop are applicable to models with small shocks, increments to Brow- 
nian motions, as well as large shocks, jumps with Poisson arrivals. We illustrate our approach with a series of examples. For lognormal models we derive shock exposure elasticities which coincide with impulse response functions familiar from the VAR literature. In more complicated models, our methodology allows us to characterize nonlinearities in the dynamics of asset prices. We contrast the habit formation models of Campbell and Cochrane (1999) and Santos and Veronesi (2008), and we document important differences in the risk price elasticities across investment horizons. We also derive elasticities for a model with recursive utility in the spirit of Hansen et al. (2008), and for a model with jump risk where the state variable evolves as a finite state Markov chain.

\section{Markov pricing with Brownian information struc- tures}

We follow the construction in Hansen and Scheinkman (2009a,b) and Hansen (2009). Consider a Markov diffusion that solves:

$$
d X_{t}=\mu\left(X_{t}\right) d t+\sigma\left(X_{t}\right) d W_{t}
$$

where $W$ is a multivariate standard Brownian motion. In this model nonlinearity is captured by the specification of $\mu$ and $\sigma$. While the state variable $X$ may well be stationary, we will use it as a building block for processes that grow or decay over time.

\subsection{Growth and discounting}

In econometric practice we often build models for the logarithms of processes. An example of such a model is

$$
A_{t}=\int_{0}^{t} \beta\left(X_{u}\right) d u+\int_{0}^{t} \alpha\left(X_{u}\right) \cdot d W_{u} .
$$

We call the resulting process, denoted by $A$, an additive functional because it depends entirely on the underlying Markov process and it is constructed by integrating over the time scale. Nonlinearity may be present in the specification of $\beta$ and $\alpha$.

While it is convenient to take logarithms when building time series models, to represent values and prices it is necessary to study levels instead of logarithms. Thus to represent growth or decay, we use the exponential of an additive functional, $M_{t}=\exp \left(A_{t}\right)$. We will refer to $M$ as a multiplicative functional parameterized by $(\beta, \alpha)$. Ito's Lemma guarantees 
that the local mean of $M$ is

$$
M_{t}\left[\beta\left(X_{t}\right)+\frac{\left|\alpha\left(X_{t}\right)\right|^{2}}{2}\right] .
$$

The multiplicative functional is a local martingale if its local mean is zero:

$$
\beta\left(X_{t}\right)+\frac{\left|\alpha\left(X_{t}\right)\right|^{2}}{2}=0
$$

There are two types of multiplicative functionals that we feature: we use one to represent stochastic growth and another for decay. For future reference, let $G$ be a stochastic growth functional parameterized by $\left(\beta_{g}, \alpha_{g}\right)$. The second will be a stochastic discount functional $S$ parameterized by $\left(\beta_{s}, \alpha_{s}\right)$. The stochastic growth functional grows exponentially over time and the stochastic discount functional decays exponentially.

\subsection{Perturbations}

To compute elasticities we construct perturbations to multiplicative functionals. A perturbation to $M$ is $M H(\mathbf{r})$ where we parameterize $H(\mathbf{r})$ using a pair $\left(\beta_{h}(x, \mathbf{r}), \mathbf{r} \alpha_{d}(x)\right)$ with $\beta_{h}(x, 0)=0$. The function $\alpha_{d}$ defines the direction of risk exposure. Thus

$$
\log H_{t}(\mathbf{r})=\int_{0}^{t} \beta_{h}\left(X_{u}, \mathrm{r}\right) d u+\mathrm{r} \int_{0}^{t} \alpha_{d}\left(X_{u}\right) \cdot d W_{u}
$$

As $r$ declines to zero, the perturbed process $M H(r)$ collapses to $M$. Let

$$
\beta_{d}(x)=\left.\frac{d}{d r} \beta_{h}(x, r)\right|_{r=0}
$$

and consider the additive functional:

$$
D_{t}=\int_{0}^{t} \beta_{d}\left(X_{u}\right) d u+\int_{0}^{t} \alpha_{d}\left(X_{u}\right) \cdot d W_{u} .
$$

We use this additive functional to represent the derivative:

$$
\left.\frac{d}{d \mathbf{r}} \log E\left[M_{t} H_{t}(\mathrm{r}) \mid X_{0}=x\right]\right|_{\mathrm{r}=0}=\frac{E\left[M_{t} D_{t} \mid X_{0}=x\right]}{E\left[M_{t} \mid X_{0}=x\right]} .
$$

See Hansen and Scheinkman (2009b) for a formal derivation including certain regularity conditions that justify this formula. Interestingly, formula (1) gives an additive decomposition of the derivative using the additive functional $D$.

In what follows we will build an alternative formula for the derivative in (1). This will 
require two steps. First we build a factorization of the multiplicative functional, and then we construct a nonlinear moving-average representation for a particular function of the Markov state.

\section{Factorization}

We obtain an alternative and convenient representation of (1) by applying a change of measure as in Hansen and Scheinkman (2009a). They provide sufficient conditions for the existence of a factorization of a multiplicative process $M$ :

$$
M_{t}=\exp (\eta t) \hat{M}_{t} \frac{e\left(X_{0}\right)}{e\left(X_{t}\right)}
$$

where $\hat{M}$ is a multiplicative martingale and $e$ is a strictly positive, smooth function of the Markov state. The parameter $\eta$ is a long-term growth or decay rate. We use the martingale $\hat{M}$ to define a new probability measure $\hat{\bullet}$ on the original probability space. The multiplicative property of $\hat{M}$ insures that $X$ remains Markovian in the new probability space. While this factorization may not be unique, there is only one such factorization in which the change in measure imposes stochastic stability and preserves the Markov structure.

This factorization is distinct from that of Ito and Watanabe (1965). The Ito and Watanabe (1965) factorization for a multiplicative supermartingale results in the product of a local martingale and decreasing functional. This factorization delivers the Markov counterpart to the risk neutral transformation used extensively in mathematical finance when it is applied to a stochastic discount factor functional. In this case the decreasing functional $M^{d}$ is

$$
M_{t}^{d}=\exp \left[-\int_{0}^{t} \rho\left(X_{u}\right) d u\right]
$$

where $\rho$ is the instantaneous interest rate. State dependence in the decreasing component makes it less valuable as a device to characterize risk price dynamics because even locally deterministic variation in instantaneous interest rates induces risk adjustments for cash flows over finite time intervals. This leads us instead to extract a long-term growth or discount rate $\eta$ as in $(2)$.

If $M$ is parameterized by $\left(\beta_{m}, \alpha_{m}\right)$, Girsanov's Theorem assures that the increment $d W_{t}$ can be written as:

$$
d W_{t}=\left[\alpha_{m}\left(X_{t}\right)+\nu\left(X_{t}\right)\right] d t+d \hat{W}_{t}
$$


Here $\nu$ is the exposure of $\log e(x)$ to $d W_{t}$ :

$$
\nu=\sigma^{\prime}\left[\frac{\partial \log e}{\partial x}\right]
$$

and $\hat{W}$ is a Brownian motion under the alternative probability measure $\hat{\ominus}$

To use this factorization in practice, we must compute $e$ and $\eta$. Hansen and Scheinkman (2009a) show how to accomplish this. Solve

$$
E\left[M_{t} e\left(X_{t}\right) \mid X_{0}=x\right]=\exp (\eta t) e(x)
$$

for any $t$ where $e$ is strictly positive. This is a (principal) eigenfunction problem, and since it holds for any $t$, it can be localized by computing

$$
\lim _{t \rightarrow 0} \frac{E\left[M_{t} e\left(X_{t}\right) \mid X_{0}=x\right]-\exp (\eta t) e(x)}{t}=0
$$

which gives an equation in $e$ and $\eta$ to be solved. The local counterpart to this equation is

$$
\mathbb{B} e=\eta e
$$

where

$$
\mathbb{B} e(x)=\left.\frac{d}{d t} E\left[M_{t} e\left(X_{t}\right) \mid X_{0}=x\right]\right|_{t=0}
$$

It can be shown that for a diffusion model, if $f$ is smooth,

$$
\mathbb{B} f=\left(\beta_{m}+\frac{1}{2}\left|\alpha_{m}\right|^{2}\right) f+\left(\sigma \alpha_{m}+\mu\right) \cdot \frac{\partial f}{\partial x}+\frac{1}{2} \operatorname{trace}\left(\sigma \sigma^{\prime} \frac{\partial^{2} f}{\partial x \partial x^{\prime}}\right)
$$

We illustrate this computation in two examples that we develop throughout the text into stylized economic models. The first example features lognormal dynamics commonly used in VAR analysis. The second example specifies a state variable that forms the basis of the consumption externality model of Santos and Veronesi (2008).

Example 3.1. Suppose that

$$
d X_{t}=\bar{\mu} X_{t} d t+\bar{\sigma} d W_{t}
$$

where $\bar{\mu}$ and $\bar{\sigma}$ are matrices of size $n \times n$ and $n \times k$, respectively. The multiplicative functional $M$ is parameterized by

$$
\begin{aligned}
\beta_{m}(x) & =\bar{\beta}_{m}^{\prime} x \\
\alpha_{m}(x) & =\bar{\alpha}_{m} .
\end{aligned}
$$


Conjecturing that the function $e(x)$ satisfies $\log e(x)=\lambda^{\prime} x$, equation (6) yields:

$$
\eta=\bar{\beta}_{m}^{\prime} x+\lambda^{\prime} \bar{\mu} x+\frac{1}{2}\left|\bar{\sigma}^{\prime} \lambda+\bar{\alpha}_{m}\right|^{2}
$$

Thus $\lambda=-\left(\bar{\mu}^{\prime}\right)^{-1} \bar{\beta}_{m}$. Under the change of measure,

$$
d X_{t}=\bar{\mu} X_{t} d t+\bar{\sigma}\left(\bar{\alpha}_{m}+\bar{\sigma}^{\prime} \lambda\right) d t+\bar{\sigma} d \hat{W}_{t}
$$

Consider now the second example. In this example the process for $\mathrm{X}$ is a member of Wong (1964)'s class of scalar Markov diffusions built to imply stationary densities that are in the Pearson family. ${ }^{1}$

Example 3.2. Let the univariate Markov state $X$ evolve as:

$$
d X_{t}=-\bar{\mu}_{1}\left(X_{t}-\bar{\mu}_{2}\right) d t-\bar{\sigma} X_{t} d W_{t}, \quad X_{t}>0
$$

where $\bar{\mu}_{1}, \bar{\mu}_{2}$, and $\bar{\sigma}$ are positive constants.

Rather than specifying the multiplicative functional $M$ and then calculating the factorization, we construct the multiplicative components directly as

$$
\begin{aligned}
& M_{t}=\exp (\eta t) \hat{M}_{t}\left(\frac{1+X_{t}}{1+X_{0}}\right) \\
& \hat{M}_{t}=\exp \left[-\frac{1}{2}\left(\hat{\alpha}_{m}\right)^{2} t+\hat{\alpha}_{m}\left(W_{t}-W_{0}\right)\right]
\end{aligned}
$$

where $\hat{\alpha}_{m}$ is a constant.

Then formula (3) implies that the evolution of $X$ under the change of measure is given by

$$
d X_{t}=-\left[\bar{\sigma} \hat{\alpha}_{m} X_{t}+\bar{\mu}_{1}\left(X_{t}-\bar{\mu}_{2}\right)\right] d t-\bar{\sigma} X_{t} d \hat{W}_{t}
$$

and the risk exposure for $\log M$ is

$$
\alpha_{m}(x)=\hat{\alpha}_{m}-\bar{\sigma} \frac{x}{1+x} .
$$

By construction, the eigenfunction is $e(x)=(1+x)^{-1}$ with eigenvalue $\eta$.

\footnotetext{
${ }^{1}$ See process $\mathrm{F}$ in Wong (1964).
} 
We use the alternative probability measure to absorb the martingale component of the multiplicative functional in our formula (2). The derivative of interest is:

$$
\left.\frac{d}{d \mathrm{r}} \log E\left[M_{t} H_{t}(\mathrm{r}) \mid X_{0}=x\right]\right|_{r=0}=\frac{\hat{E}\left[\hat{e}\left(X_{t}\right) D_{t} \mid X_{0}=x\right]}{\hat{E}\left[\hat{e}\left(X_{t}\right) \mid X_{0}=x\right]}
$$

where $\hat{e}=\frac{1}{e}$. Moreover,

$$
D_{t}=\int_{0}^{t} \beta_{d}\left(X_{u}\right) d u+\int_{0}^{t} \alpha_{d}\left(X_{u}\right) \cdot\left[\alpha_{m}\left(X_{u}\right)+\nu\left(X_{u}\right)\right] d u+\int_{0}^{t} \alpha_{d}\left(X_{u}\right) \cdot d \hat{W}_{u}
$$

For our analysis, we will seek a related representation without resort to stochastic integrals. Prior to achieving this, we will present a nonlinear moving-average representation for $\hat{e}(X)$.

\section{Nonlinear moving-average representation}

We build a nonlinear moving-average representation for a particular function of the Markov state. This formula can be viewed as a special case of the Haussmann-Clark-Ocone formula that holds under additional smoothness conditions. For example see Haussmann (1979). Let $\mathrm{T}_{\tau}$ denote the conditional expectation operator under the change in probability measure over an interval of time $\tau$. The process $\left\{\mathrm{T}_{t-u} \hat{e}\left(X_{u}\right): 0 \leq u \leq t\right\}$ is a martingale since

$$
\mathrm{T}_{t-u} \hat{e}\left(X_{u}\right)=\hat{E}\left[\hat{e}\left(X_{t}\right) \mid \mathcal{F}_{u}\right]
$$

where $\mathcal{F}_{u}$ is the $\sigma$-algebra generated by the Brownian motion until date $u$. This martingale can be represented as a stochastic integral against the Brownian motion, and, in particular, there exists an adapted process $R$ such that:

$$
\hat{e}\left(X_{t}\right)=\int_{0}^{t} R_{u} \cdot d \hat{W}_{u}+\mathrm{T}_{t} \hat{e}\left(X_{0}\right)
$$

The following assumption and our Markov specification allows us to characterize $R_{u}$.

Assumption 4.1. $\mathrm{T}_{t-u} \hat{e}(x)$ has a continuous second derivative with respect to $x$ and $a$ continuous first derivative with respect to $u$.

It is an immediate application of Ito's lemma that in this case,

$$
R_{u}=\sigma\left(X_{u}\right)^{\prime}\left[\frac{\partial}{\partial x} \mathrm{\top}_{t-u} \hat{e}\left(X_{u}\right)\right]
$$


This construction motivates a definition of a nonlinear version of an impulse response function as

$$
\sigma(x)^{\prime}\left[\frac{\partial}{\partial x} \mathrm{~T}_{t} \hat{e}(x)\right]
$$

the response of $\hat{e}\left(X_{t}\right)$ to a shock $d \hat{W}_{0} \cdot{ }^{2}$

Recall that $e$ (and hence $\hat{e}$ ) is strictly positive, and construct

$$
\begin{aligned}
\phi(x, t) & =\sigma(x)^{\prime}\left[\frac{\partial}{\partial x} \log \mathrm{T}_{t} \hat{e}(x)\right] \\
& =\frac{\sigma(x)^{\prime}\left[\frac{\partial}{\partial x} \mathrm{~T}_{t} \hat{e}(x)\right]}{\mathrm{T}_{t} \hat{e}(x)} .
\end{aligned}
$$

Note in particular that

$$
\nu(x)=-\phi(x, 0)
$$

where $\nu$ is given by (4). With this construction, we may represent (7) equivalently as:

$$
\hat{e}\left(X_{t}\right)=\int_{0}^{t}\left[\mathrm{~T}_{t-u} \hat{e}\left(X_{u}\right)\right] \phi\left(X_{u}, t-u\right) \cdot d \hat{W}_{u}+\mathrm{T}_{t} \hat{e}\left(X_{0}\right) .
$$

The function $\phi$ will play a central role in our representation of elasticities.

\subsection{Examples}

We now apply these calculations and compute the impulse response of $\hat{e}(x)$ for the lognormal example introduced earlier.

Example 4.2. Consider again Example 3.1. Recall $\hat{e}(x)=\exp \left(-\lambda^{\prime} x\right)$ for $\lambda=-\left(\bar{\mu}^{\prime}\right)^{-1} \bar{\beta}_{m}$, and conjecture that

$$
\mathrm{T}_{t} \hat{e}(x)=\exp \left[a_{0}(t)+a_{1}(t)^{\prime} x\right] .
$$

\footnotetext{
${ }^{2}$ The existing econometrics literature contains many definitions of nonlinear impulse response functions. Koop et al. (1996) and Potter (2000) examine four definitions used with linear series and assess the merits of the nonlinear analogue of each. They work in discrete time and argue that the most sensible definition is one motivated by the linear updating function used for linear series. In the Markov case their construction is based on

$$
\mathrm{T}_{t-\epsilon} \hat{e}\left(X_{\epsilon}\right)-\mathrm{T}_{t} \hat{e}\left(X_{0}\right)
$$
}

over a prediction interval $\epsilon$. In our continuous time limit, this increment becomes:

$$
\left[\frac{\partial}{\partial x} \mathrm{\top}_{t} \hat{e}\left(X_{0}\right)\right]^{\prime} \sigma\left(X_{0}\right) d \hat{W}_{0}
$$

where we take the vector multiplying the shock $d \hat{W}_{0}$ as the state-dependent impulse response. 
Under this conjecture, $a_{0}(0)=0, a_{1}(0)=-\lambda$, and

$$
\frac{d}{d t} \mathrm{~T}_{t} \hat{e}(x)=\exp \left[a_{0}(t)+a_{1}(t)^{\prime} x\right]\left[\frac{d a_{0}(t)}{d t}+\left(\frac{d a_{1}(t)}{d t}\right)^{\prime} x\right] \text {. }
$$

Alternatively, from Ito's Lemma the drift of $\mathrm{T}_{t} \hat{e}(x)$ is given by:

$$
\frac{d}{d t} \mathrm{~T}_{t} \hat{e}(x)=\exp \left[a_{0}(t)+a_{1}(t)^{\prime} x\right]\left[a_{1}(t)^{\prime} \bar{\mu} x+a_{1}(t)^{\prime} \bar{\sigma}\left(\bar{\alpha}_{m}+\bar{\sigma}^{\prime} \lambda\right)+\frac{1}{2} a_{1}(t)^{\prime} \bar{\sigma} \bar{\sigma}^{\prime} a_{1}(t)\right]
$$

Equating (9) and (10) gives

$$
\begin{aligned}
& a_{1}(t)=-\exp \left(\bar{\mu}^{\prime} t\right) \lambda \\
& a_{0}(t)=\int_{0}^{t}\left\{\frac{1}{2} \lambda^{\prime} \exp (\bar{\mu} u) \bar{\sigma} \bar{\sigma}^{\prime} \exp \left(\bar{\mu}^{\prime} u\right) \lambda-\lambda^{\prime} \exp (\bar{\mu} t) \bar{\sigma}\left(\bar{\alpha}_{m}+\bar{\sigma}^{\prime} \lambda\right)\right\} d u
\end{aligned}
$$

Thus,

$$
\phi(x, u)=\bar{\sigma}^{\prime} \exp \left(\bar{\mu}^{\prime} u\right)\left(\bar{\mu}^{\prime}\right)^{-1} \bar{\beta}_{m}
$$

since the nonlinear moving-average coefficient for $\hat{e}(x)$ is:

$$
\mathrm{T}_{t} \hat{e}(x) \phi(x, t)=\exp \left[a_{0}(t)+a_{1}(t)^{\prime} x\right] \bar{\sigma}^{\prime} \exp \left(\bar{\mu}^{\prime} t\right)\left(\bar{\mu}^{\prime}\right)^{-1} \bar{\beta}_{m}
$$

To further illustrate these methods, let us develop Example 3.2.

Example 4.3. For notational simplicity rewrite the evolution of $X$ under the change of measure as

$$
\begin{aligned}
d X_{t} & =-\hat{\mu}_{1}\left(X_{t}-\hat{\mu}_{2}\right) d t-\bar{\sigma} X_{t} d \hat{W}_{t} . \\
\hat{\mu}_{1} & =\bar{\mu}_{1}+\bar{\sigma} \hat{\alpha}_{m} \\
\hat{\mu}_{2} & =\frac{\bar{\mu}_{1} \bar{\mu}_{2}}{\hat{\mu}_{1}}
\end{aligned}
$$

Use the distorted evolution equation and expression for $\hat{e}(x)$ to calculate

$$
\mathrm{T}_{u} \hat{e}(x)=1+\hat{\mu}_{2}+\exp \left(-\hat{\mu}_{1} u\right)\left(x-\hat{\mu}_{2}\right) .
$$

As a consequence,

$$
\phi(x, u)=-\frac{\bar{\sigma} \exp \left(-\hat{\mu}_{1} u\right)}{1+\hat{\mu}_{2}+\exp \left(-\hat{\mu}_{1} u\right)\left(x-\hat{\mu}_{2}\right)} x
$$




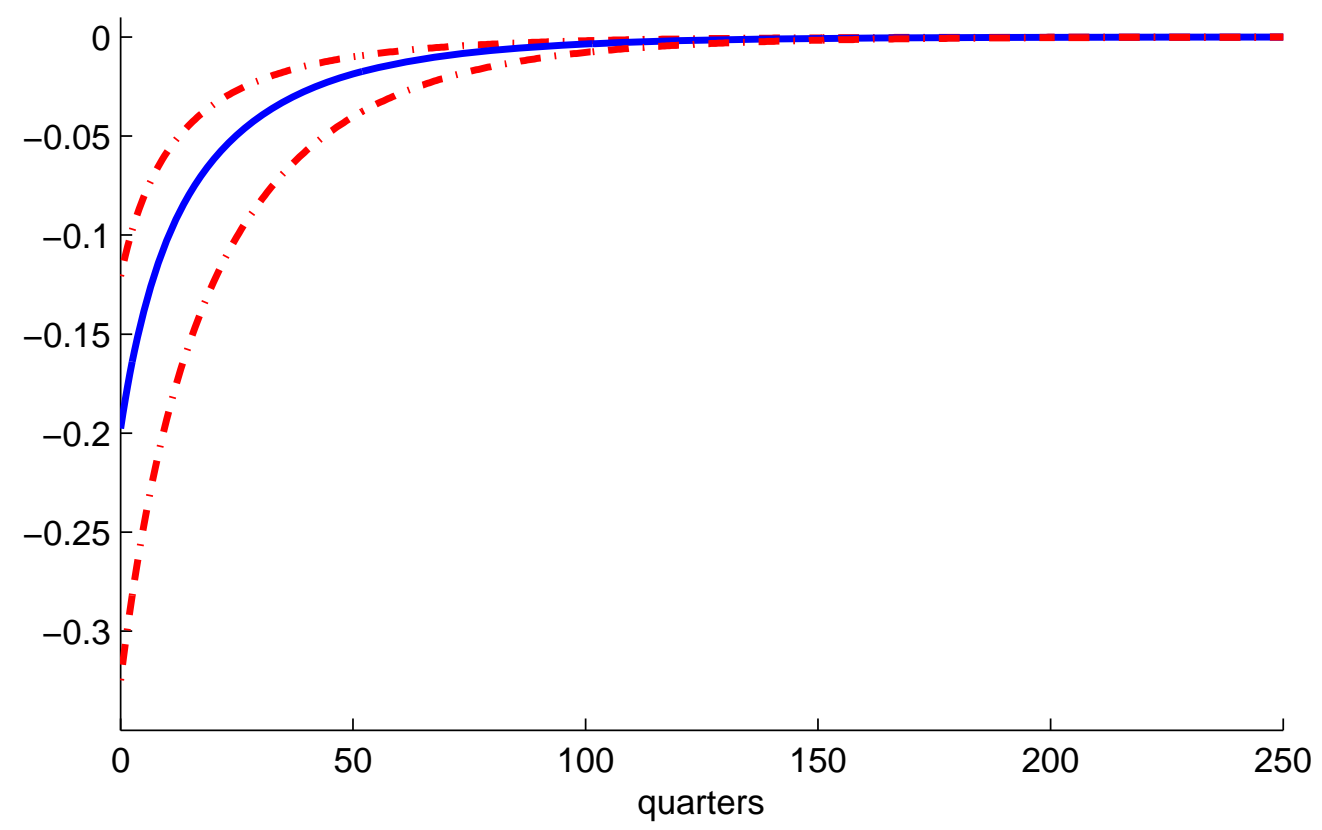

Figure 1: Plot of $\phi(x, t)$ in Example 4.3 at the $25^{\text {th }}, 50^{\text {th }}$, and $75^{\text {th }}$ quantile values of $x$. The parameterization is $\bar{\mu}_{1}=0.04, \bar{\mu}_{2}=2.28, \bar{\sigma}=0.6853, \bar{\gamma}=0.0054$.

Thus, the nonlinear moving-average representation for $\hat{e}(x)$ is

$$
\begin{aligned}
\hat{e}\left(X_{t}\right) & =\int_{0}^{t} \mathrm{~T}_{t-u} \hat{e}\left(X_{u}\right) \phi\left(X_{u}, t-u\right) d \hat{W}_{u}+1+\hat{\mu}_{2}+\exp \left(-\hat{\mu}_{1} t\right)\left(X_{0}-\hat{\mu}_{2}\right) \\
& =-\int_{0}^{t} \exp \left[-\hat{\mu}_{1}(t-u)\right] \bar{\sigma} X_{u} d \hat{W}_{u}+1+\hat{\mu}_{2}+\exp \left(-\hat{\mu}_{1} t\right)\left(X_{0}-\hat{\mu}_{2}\right)
\end{aligned}
$$

Notice that unlike in the lognormal model of Example 3.1, the function $\phi$ is state-dependent. Figure 1 displays the function at each quartile of the stationary distribution for $X$, with parameterization given in the figure.

\subsection{Malliavin derivative}

There is an alternative way to construct the nonlinear moving-average representation that is both more general and of interest in its own right. This construction is based on the Malliavin derivative, which we develop in this subsection. It is not necessary to understand this section in order to follow the remainder of our paper. We include this discussion because Malliavin differentiation is prevalent in mathematical finance. 
Consider the following perturbations to the Brownian motion between date zero and date $t$. Let $q$ be a function in $L_{2}^{n}[0, t]$ that is,

$$
\int_{0}^{t}|q(v)|^{2} d v<\infty
$$

The perturbed process is:

$$
\hat{W}_{u}+r Q_{u}, \quad 0 \leq u \leq t
$$

where $Q_{u}=\int_{0}^{u} q(v) d v$, and $r \in \mathbb{R}$. Recall that we can identify each path of a Brownian motion in $[0, t]$ with an element of $\Omega=C_{0}\left([0, t), \mathbb{R}^{n}\right)$, the set of continuous $\mathbb{R}^{n}$-valued functions starting at 0 . Given a random variable $\Phi$ defined on $\Omega$ we are interested in the derivative of $\Phi(\hat{W}+\mathrm{r} Q)$ with respect to $\mathrm{r}$. The Malliavin derivative is a process $D_{u} \Phi(\hat{W})$ that is motivated $^{3}$ by the following representation:

$$
\lim _{\mathrm{r} \rightarrow 0} \frac{\Phi(\hat{W}+\mathrm{r} Q)-\Phi(\hat{W})}{\mathrm{r}}=\int_{0}^{t} D_{u} \Phi(\hat{W}) \cdot q(u) d u .
$$

The value of the Malliavin derivative at $u$ quantifies the contribution of $d \hat{W}_{u}$ to $\Phi$. This contribution will, in general, depend on the entire Brownian path from 0 to $t$.

Fix an initial condition $x$ and a time $t$ and consider the random variable $\Phi$ defined by

$$
\Phi(\hat{W})=\hat{e}\left(X_{t}\right)
$$

where $X$ solves

$$
\begin{aligned}
d X_{u} & =\left[\mu\left(X_{u}\right)+\sigma\left(X_{u}\right)\left(\alpha\left(X_{u}\right)+\nu\left(X_{u}\right)\right)\right] d u+\sigma\left(X_{u}\right) d \hat{W}_{u} \\
& \doteq \hat{\mu}\left(X_{u}\right) d u+\sigma\left(X_{u}\right) d \hat{W}_{u} .
\end{aligned}
$$

If the functions $\hat{\mu}$ and $\sigma$ are smooth and with bounded derivatives then the random variable $X_{t}$ is the domain of the Malliavin derivative. In fact let $Y$ be the first variation process associated to $X$, that is $Y_{0}=I_{n}$ and

$$
d Y_{u}=\partial \hat{\mu}\left(X_{u}\right) Y_{u} d u+\sum_{i} \partial \sigma_{i}\left(X_{u}\right) Y_{u} d \hat{W}_{u}^{i}
$$

Here, $\partial F$ denotes the Jacobian matrix of an $\mathbb{R}^{n}$ valued function $F$ and $\sigma_{i}$ is the $i$-th column

\footnotetext{
${ }^{3}$ The construction of the Malliavin derivative usually starts by considering a subset of random variables called the Wiener polynomials and defining the Malliavin derivative using equation (12). The Malliavin derivative is then extended to a larger class of random variables using limits. Equation (12) does not necessarily hold for every random variable which has a Malliavin derivative.
} 
of the matrix $\sigma$. Then, for $u \leq t$, the $n \times n$ matrix

$$
D_{u} X_{t}=Y_{t} Y_{u}^{-1} \sigma\left(X_{u}\right)
$$

In addition, if $\hat{e}$ has bounded first derivatives, then $\Phi$ is in the domain of the Malliavin derivative and

$$
D_{u} \Phi=\nabla \hat{e}\left(X_{t}\right)^{\prime} D_{u} X_{t}
$$

where $\nabla$ is used to denote the gradient. The Haussmann-Clark-Ocone formula provides a representation of the integrator $R$ in equation (7) in terms of a Malliavin derivative: ${ }^{4}$

$$
R_{u}=\hat{E}\left[D_{u} \Phi(\hat{W}) \mid \mathcal{F}_{u}\right]
$$

and thus ${ }^{5}$

$$
\hat{e}\left(X_{t}\right)=\int_{0}^{t} \hat{E}\left[D_{u} \Phi(\hat{W}) \mid \mathcal{F}_{u}\right] \cdot d \hat{W}_{u}+\hat{E}\left[\hat{e}\left(X_{t}\right) \mid X_{0}=x\right] .
$$

Furthermore, it follows directly from equations (13)-(15) that

$$
\hat{E}\left[D_{u} \Phi(\hat{W}) \mid \mathcal{F}_{u}\right]=\hat{E}\left[D_{u} \Phi(\hat{W}) \mid X_{u}\right]
$$

When the smoothness required by Assumption 4.1 is not satisfied, we may as an alternative write the function $\phi$ via

$$
\phi(y, t-u)=\frac{\hat{E}\left[D_{u} \Phi(\hat{W}) \mid X_{u}=y\right]}{\hat{E}\left[\hat{e}\left(X_{t}\right) \mid X_{u}=y\right]}
$$

where we have initialized $X_{0}$ at $x$ and $\Phi$ depends implicitly on $t .{ }^{6}$

\section{$5 \quad$ Representing elasticities}

We now have the core ingredients for representing the elasticities that interest us. These ingredients include:

i) the coefficient $\alpha_{m}$ used in the construction of the multiplicative functional $M$;

\footnotetext{
${ }^{4}$ For a statement of this formula and the results concerning the Malliavin derivative of functions of a Markov diffusion see, for instance, Fournié et al. (1999), pages 395 and 396.

${ }^{5}$ Haussmann (1979) gives formulas for Markov dynamics for more general functions $\Phi$.

${ }^{6}$ Gourieroux and Jasiak (2005) suggest basing impulse response functions on the pathwise contribution to changing a shock at a given date. This leads them to explore more general distributional consequences of a shock. The Malliavin derivative is the continuous-time counterpart and depends on the entire shock process up to date $t$.
} 
ii) the coefficients $\beta_{d}$ and $\alpha_{d}$ used in the construction of the additive functional $D$;

iii) a change of probability measure and function ê from factorization (2);

iv) the function $\phi(x, t)$ constructed from the state-dependent coefficients in a movingaverage representation for $\hat{e}\left(X_{t}\right)$ given in (16).

The integral representation of the logarithmic derivative is given by:

Proposition 5.1. (Hansen and Scheinkman (2009b))

$$
\begin{aligned}
\left.\frac{d}{d \mathbf{r}} \log E\left[M_{t} H_{t}(\mathbf{r}) \mid X_{0}=x\right]\right|_{\mathbf{r}=0}= \\
=\frac{\hat{E}\left[\hat{e}\left(X_{t}\right) \int_{0}^{t}\left(\beta_{d}\left(X_{u}\right)+\alpha_{d}\left(X_{u}\right) \cdot\left[\alpha_{m}\left(X_{u}\right)+\phi\left(X_{u}, t-u\right)-\phi\left(X_{u}, 0\right)\right]\right) d u \mid X_{0}=x\right]}{\hat{E}\left[\hat{e}\left(X_{t}\right) \mid X_{0}=x\right]} .
\end{aligned}
$$

Exchanging orders of integration, the date $u$ contribution to the integrand is:

$$
\frac{\hat{E}\left[\hat{e}\left(X_{t}\right) \psi\left(X_{u}, t-u\right) \mid X_{0}=x\right]}{\hat{E}\left[\hat{e}\left(X_{t}\right) \mid X_{0}=x\right]}
$$

where $\hat{e}\left(X_{t}\right)$ is an extra weighting function and

$$
\psi(x, \tau) \doteq \beta_{d}(x)+\alpha_{d}(x) \cdot\left[\alpha_{m}(x)+\phi(x, \tau)-\phi(x, 0)\right] .
$$

In formula (17), $\alpha_{d}$ parameterizes the local exposure to risk that is being explored and $\beta_{d}$ is determined as a consequence of the the nature of the perturbation. The coefficient $\alpha_{m}$ is the local exposure to risk of the baseline multiplicative functional. To interpret the logarithmic derivative as an elasticity, we restrict $\left|\alpha_{d}\left(X_{t}\right)\right|^{2}$ to have a unit expectation in order that $\alpha_{d} \cdot d W_{t}$ has a unit standard deviation. The dependence of $\psi$ on the horizon to which the perturbation influences, that is the dependence on $\tau$, is only manifested in the function $\phi$.

The function $\psi$ captures the impact of the shock that occurs in the next instant. The impact of a shock at a future date will be realized through a distorted conditional expectation:

$$
\frac{\hat{E}\left[\hat{e}\left(X_{u+\tau}\right) \psi\left(X_{u}, \tau\right) \mid X_{0}=x\right]}{\hat{E}\left[\hat{e}\left(X_{u+\tau}\right) \mid X_{0}=x\right]}
$$

for $u \geq 0$ and $\tau \geq 0$. Specifically, this formula captures the date zero impact of a shock at date $u$ on the logarithmic derivative for date $u+\tau=t$. Since the process $X$ is stochastically 
stable under the change of measure, the limiting version of formula (18) as the shock date $t$ is shifted off to the future is

$$
\frac{\hat{E}\left[\hat{e}\left(X_{u+\tau}\right) \psi\left(X_{u}, \tau\right)\right]}{\hat{E}\left[\hat{e}\left(X_{u+\tau}\right)\right]}
$$

which is independent of $u$ but continues to depend on $\tau$.

We now add some structure to perturbations in order to produce formulas for $\beta_{d}$.

\subsection{Martingale perturbations}

Suppose that

$$
\beta_{h}(x, r)=-\frac{1}{2} r^{2}\left|\alpha_{d}(x)\right|^{2}
$$

Thus $H(r)$ is a local martingale for any $r$ and

$$
\beta_{d}=0
$$

In this case the date zero contribution is:

$$
\epsilon(x, t) \doteq \alpha_{d}(x) \cdot\left[\alpha_{m}(x)+\phi(x, t)-\phi(x, 0)\right]
$$

which we refer to as a shock elasticity function (of $M$ in the direction $\alpha_{d}$ ) when viewed as a function of $t$. It gives a nonlinear counterpart to an impulse response function by characterizing the (local) impact of a shock today on the expected future values of the multiplicative functionals. When the process $X$ is stationary, this function will typically have a well defined limit given by

$$
\epsilon(x, \infty) \doteq \alpha_{d}(x) \cdot\left[\alpha_{m}(x)-\phi(x, 0)\right]
$$

This nonzero limit reflects the fact that shocks are permanent.

To see the connection between our elasticity and an impulse response function, consider again Example 3.1.

Example 5.2. Use $\phi(x, t)$ as computed in Example 4.2 in equation (20) to get the shock elasticity function:

$$
\epsilon(x, t)=\bar{\alpha}_{d} \cdot\left(\bar{\alpha}_{m}-\bar{\sigma}^{\prime}\left[I-\exp \left(\bar{\mu}^{\prime} t\right)\right]\left(\bar{\mu}^{\prime}\right)^{-1} \bar{\beta}\right)
$$

This coincides with the impulse response function for $A=\log M$ where the vector $\bar{\alpha}_{d}$ selects the shock combination of interest.

In this example the shock elasticity function is not state dependent, but this outcome 
is special. Nonlinearity in the growth rate or stochastic volatility alter this conclusion. We analyze such examples in Section 6.

\subsection{Pricing growth-rate risk}

Following Hansen et al. (2008), Hansen and Scheinkman (2009a), and Hansen (2009) we consider the pricing of exposure to growth-rate risk. We study the pricing of cash flows that are multiplicative martingales in order to feature the pricing dynamics. We investigate the pricing of what is sometimes referred to as zero coupon equity (see Wachter (2005) or Lettau and Wachter (2007)), that is a claim to a single random payoff at a point in time $t$.

To feature price dynamics, suppose that the growth functional $G$ and each perturbation $G H(\mathrm{r})$ are multiplicative martingales:

$$
\beta_{g}(x)+\beta_{h}(x, r)=-\frac{1}{2}\left|\alpha_{g}(x)+r \alpha_{d}(x)\right|^{2} .
$$

The price of cash flow $G$ is

$$
E\left(S_{t} G_{t} \mid X_{0}=x\right)
$$

Since $G_{t}$ has conditional expectation equal to one,

$$
-\frac{1}{t} \log E\left(S_{t} G_{t} \mid X_{0}=x\right)
$$

is the expected rate of return.

Given a direction $\alpha_{d}$, construct the additive functional:

$$
D_{t}=-\int_{0}^{t} \alpha_{d}\left(X_{u}\right) \cdot \alpha_{g}\left(X_{u}\right) d u+\int_{0}^{t} \alpha_{d}\left(X_{u}\right) \cdot d W_{u}
$$

Then the growth-rate risk price for direction $\alpha_{d}$ is defined to be the marginal change in the negative logarithm of the price (logarithm of the expected return) with respect to the exposure to a shock $\alpha_{d}\left(X_{t}\right) \cdot d W_{t}$. Formally it is given by

$$
\rho_{t}=-\frac{\frac{1}{t} E\left(S_{t} G_{t} D_{t} \mid X_{0}=x\right)}{E\left(S_{t} G_{t} \mid X_{0}=x\right)}
$$

We take the negative because risk exposure is typically unwelcome to investors. We make $\rho_{t}$ an elasticity by normalizing the long-run riskiness of the exposure to be one per unit time. Specifically we restrict

$$
E\left[\left|\alpha_{d}\left(X_{t}\right)\right|^{2}=1\right.
$$


We decompose this risk price by applying Proposition 5.1. The shock-price elasticity function

$$
\pi(x, t)=-\alpha_{d}(x) \cdot\left[\alpha_{s}(x)+\phi(x, t)-\phi(x, 0)\right]
$$

represents the time $u=0$ contribution to the risk price. We call the price elasticities to shocks at intermediate dates $u$ risk-price increments. These intermediate contributions are of the form (18) and Proposition 5.1 shows how to write the growth-rate risk price (21) as the integral of the incremental prices over the lifetime of the cash flow.

\subsection{Alternative perturbations}

In order to focus exclusively on price elasticities, in section 5.2 we structured our perturbations so that $G H(r)$ is a martingale for each $r$. Suppose instead we follow the approach in section 5.1 , by constructing a martingale perturbation:

$$
\log H_{t}(\mathrm{r})=-\frac{\mathrm{r}^{2}}{2} \int_{0}^{t}\left|\alpha_{d}\left(X_{u}\right)\right|^{2} d u+\mathrm{r} \int_{0}^{t} \alpha_{d}\left(X_{u}\right) \cdot d W_{u}
$$

Typically $G H(r)$ will not be a martingale, and as a consequence in our study of returns we must also take account of how the perturbation alters the expected payoff.

The return of interest is given by:

$$
\frac{G_{t} H_{t}(\mathrm{r})}{E\left[S_{t} G_{t} H_{t}(\mathrm{r}) \mid X_{0}=x\right]}
$$

In our study of the dynamics of expected rates of return we have to consider contributions from both the expected payoff and from the price:

$$
\frac{1}{t} \log E\left[G_{t} H_{t}(\mathrm{r}) \mid X_{0}=x\right]-\frac{1}{t} \log E\left[S_{t} G_{t} H_{t}(\mathrm{r}) \mid X_{0}=x\right] .
$$

In light of these two contributions, we compute two elasticity functions: the shockexposure elasticity of $G$ in the direction $\alpha_{d}$ and the shock-exposure elasticity of $S G$ in the same direction. The first elasticity imitates our earlier calculation with

$$
\left.\frac{d}{d \mathrm{r}} \log E\left[G_{t} H_{t}(\mathrm{r}) \mid X_{0}=x\right]\right|_{\mathrm{r}=0}=\frac{\hat{E}_{g}\left[\int_{0}^{t} \hat{e}_{g}\left(X_{t}\right) \epsilon_{g}\left(X_{u}, t-u\right) d u \mid X_{0}=x\right]}{\hat{E}_{g}\left[\hat{e}_{g}\left(X_{t}\right) \mid X_{0}=x\right]}
$$

where

$$
\epsilon_{g}(x, \tau) \doteq \alpha_{d}(x) \cdot\left[\alpha_{g}(x)+\phi_{g}(x, \tau)-\phi_{g}(x, 0)\right]
$$


This gives the exposure contribution over an interval $t$ and its decomposition using discounted shock-exposure elasticities for shocks at intermediate dates $u$. In this formula we use the subscript $g$ because we chose $M=G$, and the elasticity measures how a shock today influences the growth functional in future time periods.

The second elasticity is entirely analogous except that $M=S G \doteq V$ and measures value responses:

$$
\frac{\hat{E}_{v}\left[\int_{0}^{t} \hat{e}_{v}\left(X_{t}\right) \epsilon_{v}\left(X_{u}, t-u\right) d u \mid X_{0}=x\right]}{\hat{E}_{v}\left[\hat{e}_{v}\left(X_{t}\right) \mid X_{0}=x\right]}
$$

where

$$
\epsilon_{v}(x, \tau) \doteq \alpha_{d}(x) \cdot\left[\alpha_{v}(x)+\phi_{v}(x, \tau)-\phi_{v}(x, 0)\right]
$$

In this formula,

$$
\alpha_{v}=\alpha_{s}+\alpha_{g}
$$

Combining these two integral contributions, we obtain a risk price elasticity that takes into account the predictability of $G$ and its perturbed counterpart:

$$
\begin{aligned}
& \frac{d}{d \mathrm{r}} \log E\left[G_{t} H_{t}(\mathrm{r}) \mid X_{0}=x\right]-\left.\log E\left[S_{t} G_{t} H_{t}(\mathrm{r}) \mid X_{0}=x\right]\right|_{\mathrm{r}=0}= \\
& \frac{\hat{E}_{g}\left[\int_{0}^{t} \hat{e}_{g}\left(X_{t}\right) \epsilon_{g}\left(X_{u}, t-u\right) d u \mid X_{0}=x\right]}{\hat{E}_{g}\left[\hat{e}_{g}\left(X_{t}\right) \mid X_{0}=x\right]}-\frac{\hat{E}_{v}\left[\int_{0}^{t} \hat{e}_{v}\left(X_{t}\right) \epsilon_{v}\left(X_{u}, t-u\right) d u \mid X_{0}=x\right]}{\hat{E}_{v}\left[\hat{e}_{v}\left(X_{t}\right) \mid X_{0}=x\right]} .
\end{aligned}
$$

From the instantaneous contribution to these integrals, we construct an alternative shockprice elasticity function:

$$
\pi(x, t)=-\alpha_{d}(x) \cdot\left[\alpha_{s}(x)+\left(\phi_{v}-\phi_{g}\right)(x, t)-\left(\phi_{v}-\phi_{g}\right)(x, 0)\right]
$$

When $G$ is a multiplicative martingale, $\phi_{g}$ is identically zero and this coincides with our previous construction of a shock price elasticity. The integral contributions, or risk-price increments, will still be different because $G$ and $V$ are associated with two different changes in measure.

\section{Example economies}

To illustrate the methods we developed, we provide the elasticity calculations for several models from the existing asset pricing literature. First we contrast the price elasticities implied by two models in which investors have preferences that reflect external habits or consumption externalities. Next we postulate consumption dynamics that contain a small 
predictable component in macroeconomic growth and stochastic volatility. We investigate how the price elasticities change when we alter the investors' preferences from a baseline power utility specification to a recursive utility counterpart.

\subsection{External habit models}

The class of external habit models includes a variety of specifications that strive to explain empirical characteristics of the asset price dynamics. One important aspect, analyzed in Santos and Veronesi (2008) and other papers, are the differences in returns on cash flows of alternative maturities. We share a similar interest and focus on the implied pricing dynamics as reflected in the term structure of shock-price elasticities. We calculate these elasticities for the models of Campbell and Cochrane (1999) and Santos and Veronesi (2008) (abbreviated as CC and SV, respectively) and highlight important differences. We start with the SV model for which there are closed-form solutions for the shock-price elasticities. For comparison we use a continuous-time version of the $\mathrm{CC}$ model and rely on numerical calculations similar to those in Wachter (2005).

Both models specify the stochastic discount factor as a multiplicative functional

$$
\begin{aligned}
S_{t} & =\exp (-\delta t)\left(\frac{C_{t}-C_{t}^{*}}{C_{0}-C_{0}^{*}}\right)^{-\gamma} \\
& =\exp (-\delta t)\left(\frac{C_{t}}{C_{0}}\right)^{-\gamma} \frac{e\left(X_{0}\right)}{e\left(X_{t}\right)} .
\end{aligned}
$$

$C^{*}$ is an external consumption reference process and $C$ is aggregate consumption, evolving as a geometric Brownian motion

$$
d \log C_{t}=\bar{\beta}_{c} d t+\bar{\alpha}_{c} d W_{t}
$$

The growth functional of interest is the aggregate consumption process itself, $G=C$.

Santos and Veronesi (2008) specify the transitory component as

$$
e\left(X_{t}\right)^{-1}=\left(1-\frac{C_{t}^{*}}{C_{t}}\right)^{-\gamma}=1+X_{t} \geq 1
$$

where the process $X$ evolves as in Example 3.2. Then $M=S C$ is a multiplicative functional of the form in Example 4.3, where $\bar{\alpha}_{m}=(1-\gamma) \bar{\alpha}_{c}$. Additionally, the loading of $X$ on the shock, $\bar{\sigma}$, is expressed as a factor of $\bar{\alpha}_{c}, \bar{\sigma}=\chi \bar{\alpha}_{c}$. The local risk price (identical to the local shock-price elasticity) is

$$
\gamma \bar{\alpha}_{c}+\chi \bar{\alpha}_{c} \frac{X_{t}}{1+X_{t}}
$$


In Campbell and Cochrane (1999), the transitory component is given by

$$
e\left(X_{t}\right)^{-1}=\exp \left(\gamma\left(X_{t}+b\right)\right)
$$

and the process $X$ follows

$$
d X_{t}=-\xi\left(X_{t}-\mu_{x}\right) d t+\lambda\left(X_{t}\right) \sigma_{c} d W_{t}
$$

with the volatility factor $\lambda(x)=1-(1+\zeta x)^{1 / 2}$ and $\zeta=2 \xi /\left(\gamma\left|\bar{\alpha}_{c}\right|^{2}\right)$. This implies the local shock-price elasticity

$$
\gamma \bar{\alpha}_{c}-\gamma \lambda(x) \bar{\alpha}_{c}=\gamma(1+\zeta x)^{1 / 2} \bar{\alpha}_{c}
$$

The SV and CC models thus amplify the local shock-price elasticities in the power utility model, $\gamma \bar{\alpha}_{c}$, by a state-dependent factor.

To facilitate comparisons between the SV and CC specifications, we fix $\gamma=2$ for both models but set the parameters of the SV model so that the distribution of local risk prices is similar to that in the CC model. Formally, the parameters $\mu_{x}$ and $\chi$ are chosen to minimize the Kullback-Leibler divergence (the log-likelihood ratio) with respect to the local risk price density of the CC model. ${ }^{7}$ Figure 2 reports the stationary densities for the local risk prices in the two models. The densities have rather different shapes even after we have adjusted the SV parameter values to make them look as similar as possible.

For simplicity we consider only the single shock case and set $\alpha_{d}$ to unity. We use formula (22) to calculate the following shock-price elasticity function, which in this example is statedependent:

$$
\pi(x, t)=\gamma \bar{\alpha}_{c}+\frac{\exp \left(-\hat{\mu}_{1} t\right)}{1+\hat{\mu}_{2}+\exp \left(-\hat{\mu}_{1} t\right)\left(x-\hat{\mu}_{2}\right)} \chi \bar{\alpha}_{c} x
$$

For the CC model we do not have quasi-analytical formulas at our disposal and instead rely on numerical methods to compute the function.

The top panel of Figure 3 displays the elasticity function for the quartiles of the stationary distribution of the state variable $X$, and the bottom panel compares with the shock-price elasticity function implied by Campbell and Cochrane (1999). The elasticity function of the SV model decays relatively quickly and is near its limiting value by about 50 quarters. ${ }^{8}$ On the other hand, that of CC remains relatively flat for 100 quarters and does not approach its

\footnotetext{
${ }^{7}$ When the original SV parameterization is used the local risk prices in CC are roughly twice as large as those of SV. For the SV specification, it is tricky to change $\gamma$. If the specification of the consumption externality is held fixed the convenient functional form for the state evolution is lost.

${ }^{8}$ This limiting value as the maturity $t \rightarrow \infty$ is equal to the elasticity from the power utility model, $\gamma \bar{\alpha}_{c}$.
} 

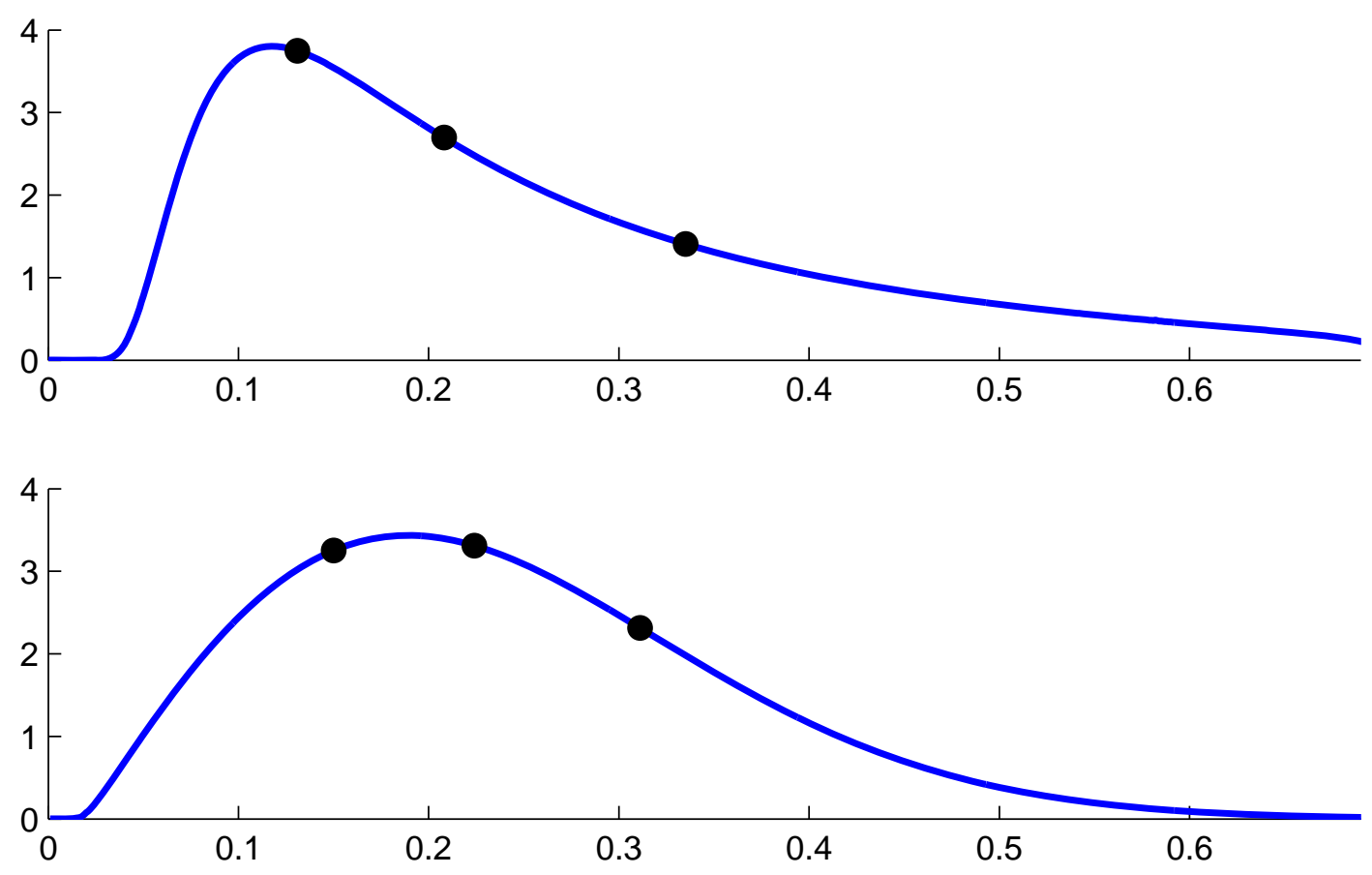

Figure 2: The top panel displays the stationary density of local risk prices in the Santos and Veronesi $(2008)$ model. The $25^{\text {th }}, 50^{\text {th }}$, and $75^{\text {th }}$ quantiles are marked with circles. The parameterization is $\chi=126.9, \mu_{1}=0.04, \mu_{2}=2.280, \bar{\alpha}_{c}=0.0054, \gamma=2$. The bottom panel compares with the model of Campbell and Cochrane (1999) as outlined in Hansen (2009) with parameter values $\xi=0.035, \mu_{x}=0.4992, \bar{\alpha}_{c}=0.0054$, and $\gamma=2$.

limiting value until about 300 quarters. Thus, the SV model implies a much less persistent impact of exposure to a current shock on the prices of cash flows further in the future.

Recall that the shock-price elasticities depict the impact for valuation of shock exposure that occurs over the next instant. We now shift forward the date of the exposure to be $u$ periods into the future. This gives the risk-price increments which are a distorted conditional expectation of the shock-price elasticity function reported in Figure 3:

$$
\begin{aligned}
& -\frac{\hat{E}\left[\hat{e}\left(X_{u+\tau}\right)\left[\alpha_{s}\left(X_{u}\right)+\phi\left(X_{u}, \tau\right)-\phi\left(X_{u}, 0\right)\right] \mid X_{0}=x\right]}{\hat{E}\left[\hat{e}\left(X_{u+\tau}\right) \mid X_{0}=x\right]} \\
& =\gamma \bar{\alpha}_{c}+\exp \left(-\hat{\mu}_{1} \tau\right) \chi \frac{\hat{\mu}_{2}+\exp \left(-\hat{\mu}_{1} u\right)\left(x-\hat{\mu}_{2}\right)}{1+\hat{\mu}_{2}+\exp \left(-\hat{\mu}_{1}(u+\tau)\right)\left(x-\hat{\mu}_{2}\right)} \bar{\alpha}_{c}
\end{aligned}
$$

where $u+\tau=t$ is the investment horizon. These curves (indexed by $u$ ) have a well defined 

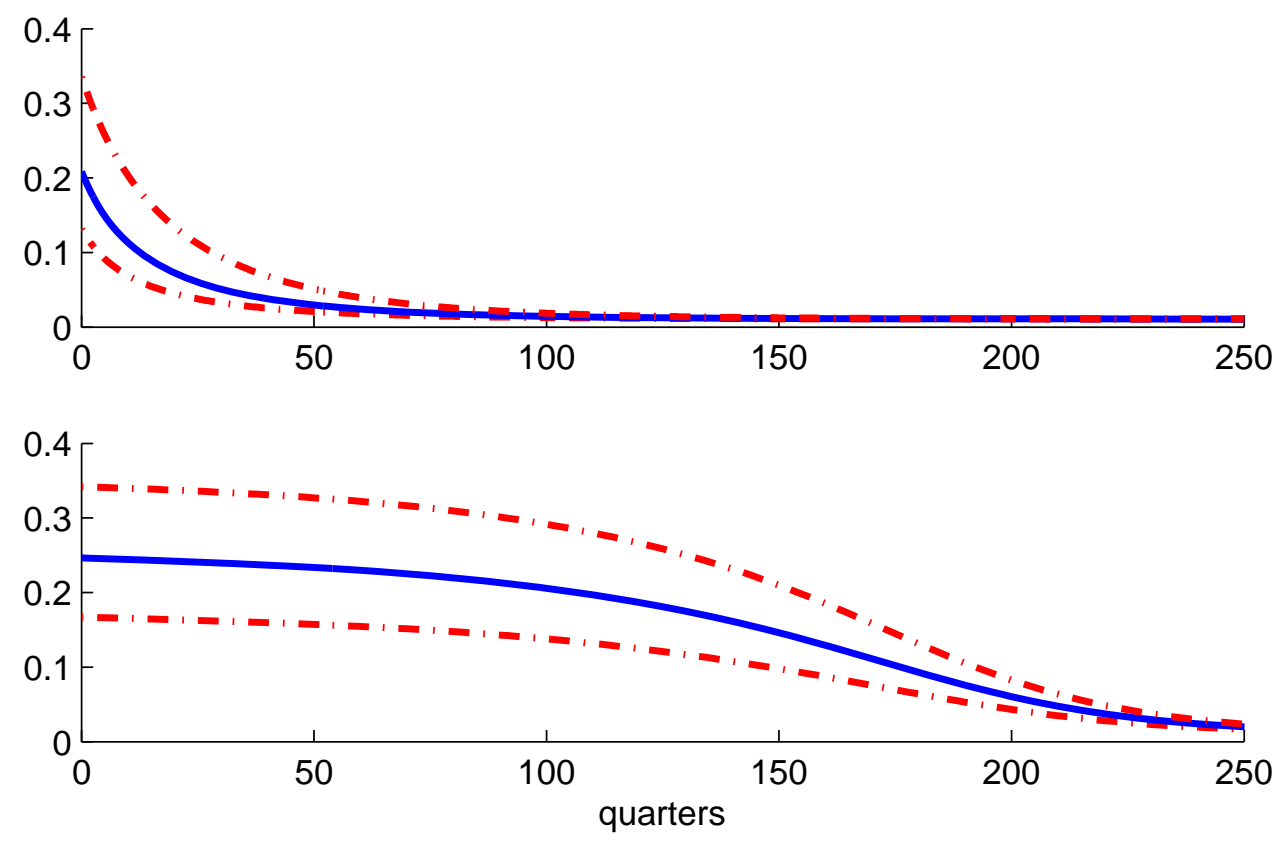

Figure 3: The top panel displays the shock-price elasticity function in the Santos and Veronesi (2008) model, while the bottom panel compares with the Campbell and Cochrane (1999) model. The solid curve conditions on the median state, while the dot-dashed curves condition on the $25^{\text {th }}$ and $75^{\text {th }}$ quantiles. Both parameterizations are as in Figure 2.

limit as $u \rightarrow \infty$ given by formula (19), which in the case of the SV model is

$$
\gamma \bar{\alpha}_{c}+\exp \left(-\hat{\mu}_{1} \tau\right) \frac{\hat{\mu}_{2}}{1+\hat{\mu}_{2}} \chi \bar{\alpha}_{c}
$$

For the CC model counterpart we again rely on numerical calculations.

Figure 4 compares the limiting shock-price elasticities in the SV and CC models. The upper panel shows that in Santos and Veronesi (2008) the limiting shock-price elasticity decays exponentially, and it sits somewhat higher than the local elasticity conditioned on the upper quartile. This upward pull is due to the heaviness of the upper tail of the stationary distribution of $X$.

The bottom panel shows that in the CC model, this upward pull on the limiting elasticity is extreme - the limiting local contribution is higher by a factor of 30 compared to the local elasticity at the median state. ${ }^{9}$ The limiting elasticity curves sharply contrast what the SV

\footnotetext{
${ }^{9}$ To elucidate the calculation, consider the numerator of the limiting contribution in formula (19) for $\tau=0: \hat{E}\left[\hat{e}\left(X_{u}\right) \pi\left(X_{u}, 0\right)\right]=-\int \hat{q}(x) \hat{e}(x) \alpha_{m}(x) d x$ where $\hat{q}(x)$ denotes the stationary density for the state variable under the change of measure. Hansen (2009) shows that the large $x$ approximation of $\hat{q}(x) \hat{e}(x)$ is $\exp (-k \sqrt{x})$ with a small coefficient $k$ while $-\alpha_{m}(x)$ behaves as $\sqrt{x}$ for large $x$. The slow decay of $\hat{q}(x) \hat{e}(x)$ combined with the unboundedness of the local risk price function leads to the high limiting contributions displayed in the bottom panel of Figure 4.
} 

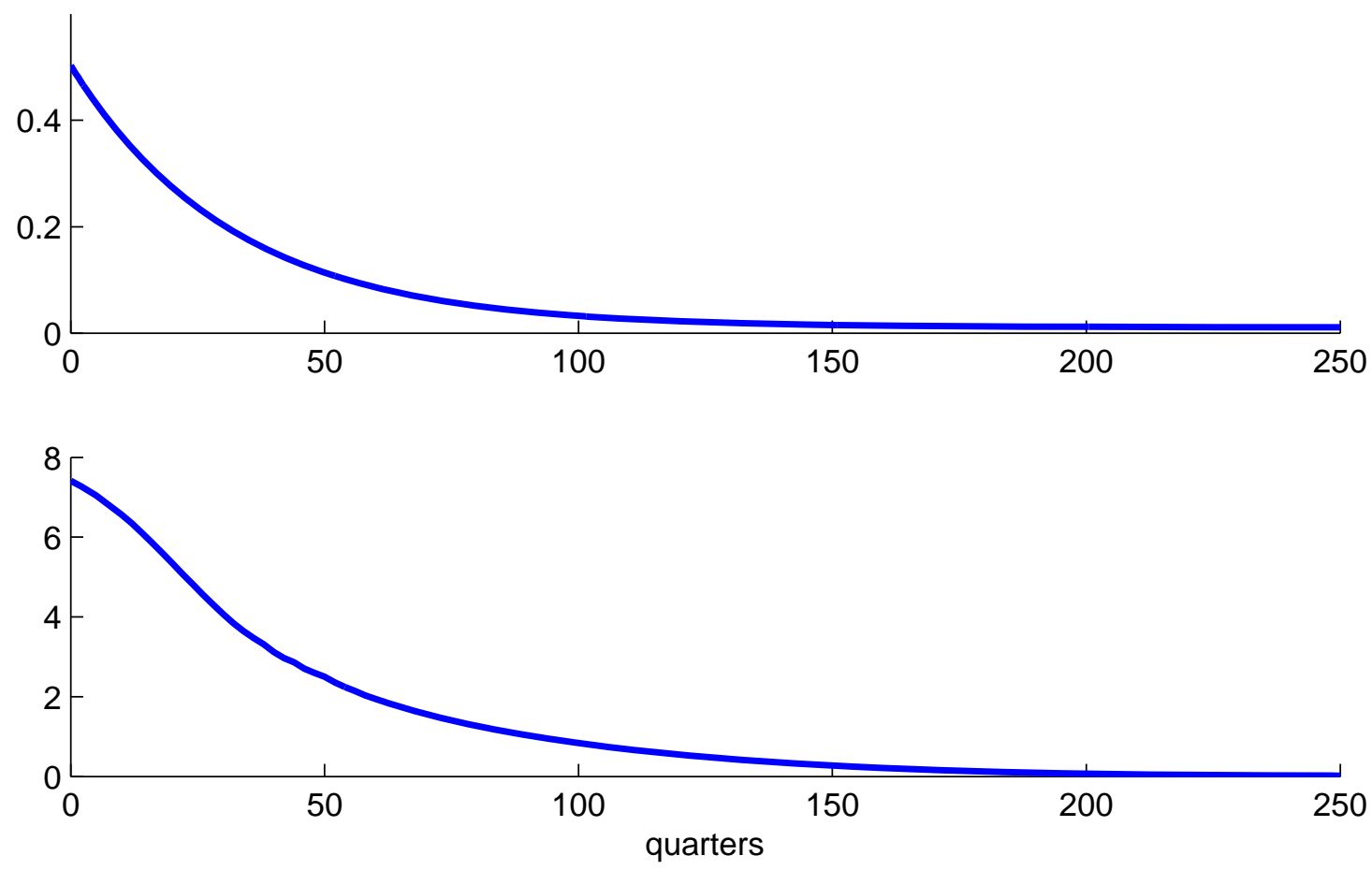

Figure 4: A comparison of the limiting shock-price elasticities of the Santos and Veronesi (2008) model (top panel) with the Campbell and Cochrane (1999) model (bottom panel). Both parameterizations are as in Figure 2.

and CC models imply about how tail risk affects the prices of cash flows with long maturities.

So far, we have analyzed the shock-price elasticity $\pi(x, t)$ and its limiting counterpart. We now consider pricing growth rate risk as in Hansen and Scheinkman (2009a) and Hansen (2009) where we parameterize the exposure to risk to occur over the entire investment horizon. These growth-rate risk prices are integrals of the price elasticities discussed previously as depicted in Section 5.1 and scaled by the investment horizon $t$. Hansen (2009) discusses the computation of the risk prices in more detail.

We plot the risk prices for the two models in Figure 5 as functions of the investment horizon. The top panel shows that the risk prices in the SV model show a similar decaying pattern as the shock-price elasticities. The decay rate for the risk prices is slower relative to the elasticity function because risk prices aggregate the elasticity contributions at intermediate shock dates. In the $\mathrm{CC}$ model, the growth-rate risk prices increase with maturity up until about 200 quarters. This is consistent with the dramatic upward shift in the shock-price elasticity function for the $\mathrm{CC}$ model as we move forward the exposure date, thus approaching the limit curve depicted in Figure 4. It is only after 200 quarters that the growth-rate risk prices start to decrease. Thus the risk price dynamics are very different for the SV and CC 

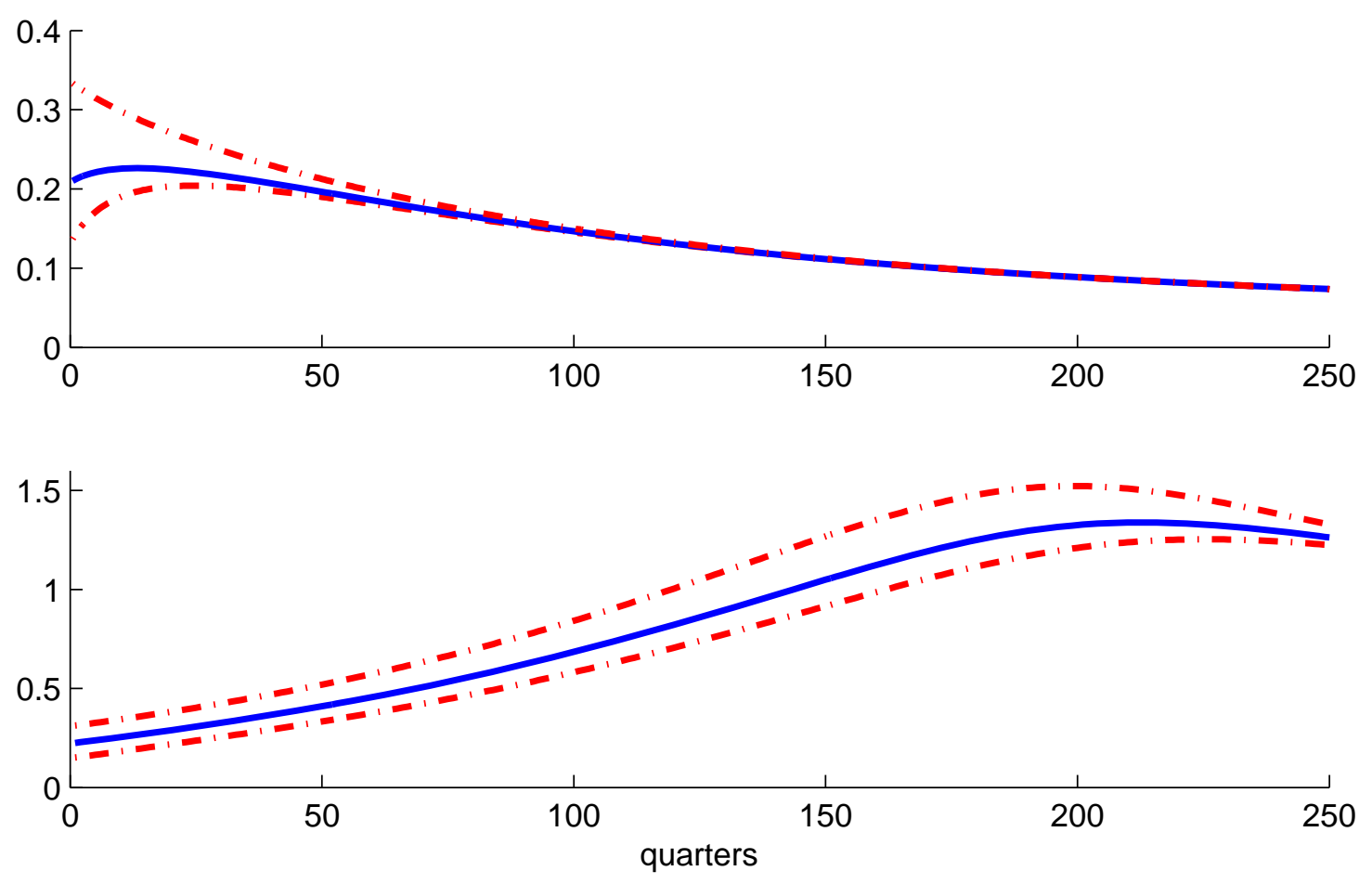

Figure 5: The top panel displays risk prices as a function of investment horizon in the Santos and Veronesi (2008) model. The solid curve conditions on the median state, while the dotdashed curves condition on the $25^{\text {th }}$ and $75^{\text {th }}$ quantiles. The bottom panel compares with the Campbell and Cochrane (1999) model. Both parameterizations are as in Figure 2.

models even though they were designed to capture similar empirical phenomenon, larger risk prices in bad times than good times.

\subsection{Breeden-Lucas and Epstein-Zin preferences}

The literature on "long-run risk" features models with a small predictable component in the growth rate of consumption and investors endowed with recursive utility preferences for which the intertemporal composition of risk matters. Stochastic volatility in the macroeconomy is included in part as a mechanism for risk prices to fluctuate over time. Hansen et al. (2007) and Hansen (2009) present an example that is the continuous-time counterpart to the model of Bansal and Yaron (2004). This example generalizes the log-normal dynamics introduced in Examples 3.1 and 4.2 by the inclusion of a square root process for the evolution of macroeconomic volatility. 


\subsubsection{State dynamics}

In line with the example in Hansen (2009), we specify the dynamics for the state vector $X_{t}=\left(X_{t}^{[1]}, X_{t}^{[2]}\right)^{\prime}$ as

$$
\mu(x)=\left[\begin{array}{c}
\bar{\mu}_{1} x^{[1]} \\
\bar{\mu}_{2}\left(x^{[2]}-1\right)
\end{array}\right] \quad \sigma(x)=\sqrt{x^{[2]}}\left[\begin{array}{c}
\bar{\sigma}_{1} \\
\bar{\sigma}_{2}
\end{array}\right]
$$

and consider a multiplicative functional for consumption parameterized by

$$
\beta(x)=\bar{\beta}_{0}+\bar{\beta}_{1} x^{[1]}+\bar{\beta}_{2}\left(x^{[2]}-1\right) \quad \alpha(x)=\sqrt{x^{[2]}} \bar{\alpha} .
$$

This specification of the dynamics contains a predictable component in the multiplicative functional modeled by $X^{[1]}$, and allows for stochastic volatility modeled by the scalar variance process $X^{[2]}$. Our variance process stays strictly positive, and we prevent it from being pulled to zero by imposing the restriction $\bar{\mu}_{2}+\frac{1}{2}\left|\bar{\sigma}_{2}\right|^{2}<0$. To guarantee stationarity, assume that $\bar{\mu}_{1}$ has eigenvalues with strictly negative real parts.

\subsubsection{Investors' preferences}

We compare the shock-price elasticities for two specifications of investors' preferences. In the Breeden (1979) and Lucas (1978) specification, investors have time-separable power utility with relative risk aversion coefficient $\gamma$. In the second case, we endow investors with recursive preferences of the Kreps and Porteus (1978) or Epstein and Zin (1989) type, analyzed in continuous time by Duffie and Epstein (1992). We refer to the first model as the BL model and the second as the EZ model.

In the BL model, we immediately have the stochastic discount factor as:

$$
S_{t}=\exp (-\delta t)\left(\frac{C_{t}}{C_{0}}\right)^{-\gamma}
$$

In the EZ model the stochastic discount factor requires more calculation. Let $\left\{V_{t}\right\}$ denote the continuation value for the recursive utility specification, and denote the inverse of the elasticity of intertemporal substitution by $\varrho$. The continuous-time recursive utility evolution is restricted by:

$$
0=\frac{\delta}{1-\varrho}\left[\left(C_{t}\right)^{1-\varrho}-\left(V_{t}\right)^{1-\varrho}\right]\left(V_{t}\right)^{\varrho}+\left[\frac{\lambda_{t}}{(1-\gamma)\left(V_{t}\right)^{1-\gamma}}\right] V_{t}
$$


where $\lambda_{t}$ is the local mean:

$$
\lambda_{t}=\lim _{\epsilon \downarrow 0} \frac{E\left[\left(V_{t+\epsilon}\right)^{1-\gamma}-\left(V_{t}\right)^{1-\gamma} \mid \mathcal{F}_{t}\right]}{\epsilon} .
$$

Notice that this recursion is homogeneous of degree one in consumption and the continuation value process. The limiting version for $\varrho=1$ is given as:

$$
0=\delta\left(\log C_{t}-\log V_{t}\right) V_{t}+\left[\frac{\lambda_{t}}{(1-\gamma)\left(V_{t}\right)^{1-\gamma}}\right] V_{t}
$$

In what follows we impose the unitary elasticity of substitution restriction as a device to obtain quasi-analytical solutions. ${ }^{10}$ The stochastic discount factor is then:

$$
S_{t}=\exp (-\delta t)\left(\frac{C_{t}}{C_{0}}\right)^{-1} \tilde{S}_{t}
$$

where $\tilde{S}_{t}$ is the multiplicative martingale component of

$$
\left(\frac{V_{t}}{V_{0}}\right)^{1-\gamma}
$$

given by the Ito and Watanabe (1965) decomposition described previously. ${ }^{11}$ This martingale component inherits the forward-looking features of the continuation value process.

Hansen (2009) shows that both stochastic discount factors share the same martingale component when $\delta \searrow 0$, and thus the long-term pricing implications for both the BL and EZ models coincide in this limiting case.

Given the state dynamics, constructing this martingale component is straightforward. The necessary calculations to arrive at the stochastic discount factor are detailed in Appendix A. Appendix $\mathrm{C}$ provides an alternative derivation.

\subsubsection{Elasticities}

This model specification implies two useful properties in calculating shock elasticities. First, for a multiplicative functional parameterized by (26), the principal eigenfunction associated with the martingale decomposition is loglinear in the state variables, $e(x)=\exp \left(\lambda^{\prime} x\right)$. Note then that $\left\{e\left(X_{t}\right) / e\left(X_{0}\right): t \geq 0\right\}$ is also a multiplicative functional of form (26). Second, conditional expectations of such a multiplicative functional are loglinear in the state variables,

\footnotetext{
${ }^{10}$ The impact of the intertemporal elasticity on the risk prices vanishes as we let the subjective rate of discount approach unity.

${ }^{11}$ The martingale contribution is well known to support an interpretation of a model in which beliefs are distorted as a device to enforce a concern about model misspecification or a preference for robustness.
} 
with time-varying coefficients given as solutions to a set of first-order ordinary differential equations. See Appendix A for details.

Using the two properties of process (26) mentioned above,

$$
\mathrm{T}_{t} \hat{e}(x)=\exp \left\{\theta_{0}(t)+\theta_{1}(t) \cdot x^{[1]}+\theta_{2}(t) x^{[2]}\right\}
$$

where the coefficients $\theta(t)$ are given in Appendix A. This implies

$$
\phi(x, t)=\left[\bar{\sigma}_{1}^{\prime} \theta_{1}(t)+\bar{\sigma}_{2}^{\prime} \theta_{2}(t)\right] \sqrt{x^{[2]}}
$$

The shock-price elasticities follow, and are displayed in Figure 6 for both the BL and EZ models. The growth functional that we use is the martingale component of the multiplicative factorization (2) of consumption.

The calculation is parameterized such that the innovations to $\log C, X^{[1]}$, and $X^{[2]}$ are mutually uncorrelated. We interpret these innovations as consumption, growth-rate, and volatility shocks, although a structural model of the macro-economy would, among other things, lead to more interesting labels assigned to shocks. We plot the shock-price elasticities for the volatility shock with opposite sign because a surprise increase in volatility is bad for agents.

Since the consumption shock has only a permanent impact on consumption, the associated risk price elasticities coincide for the two utility specifications. In contrast, local elasticities for the growth-rate and volatility risk in the BL model are zero, while in the forward-looking EZ model the elasticities for arbitrarily short investment horizons remain bounded away from zero. The shock price elasticities for the BL model mirror closely the shock exposure elasticities for aggregate consumption scaled by $\gamma$. The exposure elasticities are reported in Figure 7 . This close link reflects the underlying time separability in preferences. In the EZ model exposure of future consumption to growth-rate and volatility risk induces fluctuations in the continuation utility. As a consequence both the growth-rate state and volatility state evolution directly influence the equilibrium stochastic discount factor in the EZ model with recursive utility investors. The corresponding shock-price elasticity function is close to flat for this model with the limits being essentially the same as for the BL model. ${ }^{12}$ This reflects the importance of the martingale component $\tilde{S}$ in the stochastic discount factor process. Notice that overall the shock-exposure elasticities are larger for exposure to growth rate risk than volatility risk.

\footnotetext{
${ }^{12}$ They are identical in the limiting case in which the subjective rate of discount is zero.
} 

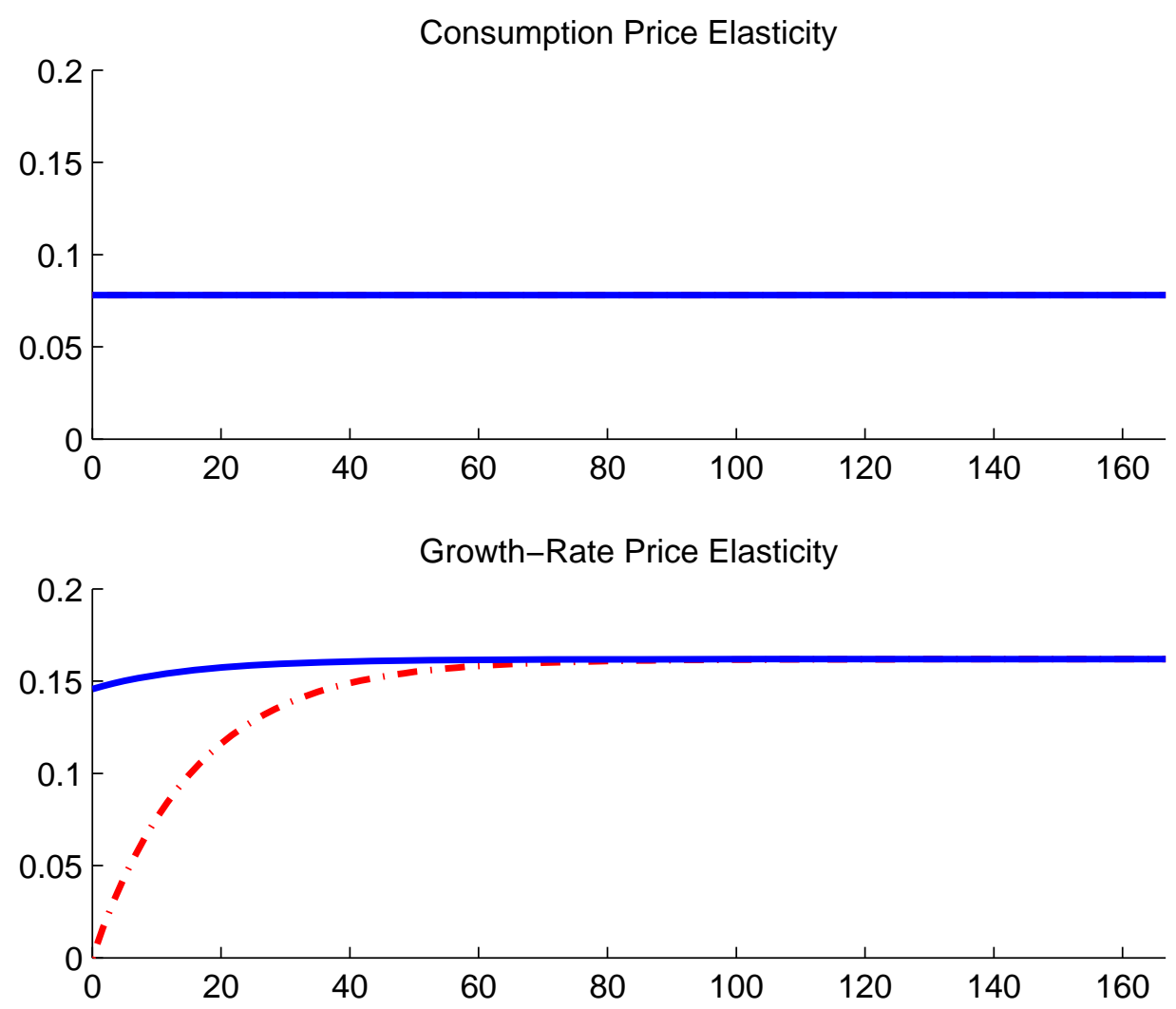

Volatility Price Elasticity

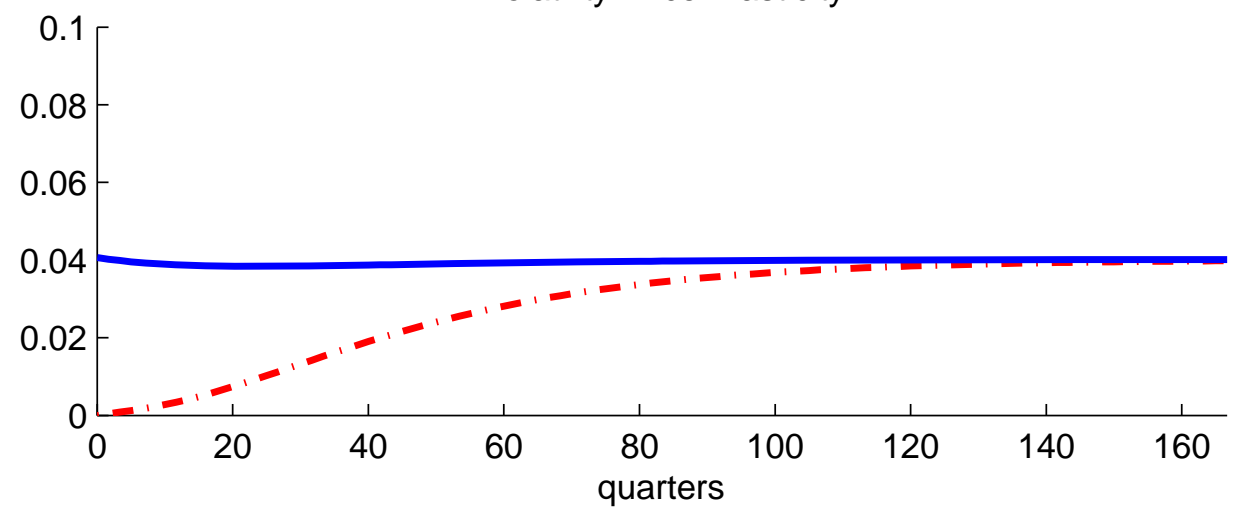

Figure 6: Shock-price elasticities under both the BL (dashed) and EZ (solid) preference specifications. The parameterization is $\bar{\beta}_{c, 0}=0.0015, \bar{\beta}_{c, 1}=1, \bar{\beta}_{c, 2}=0, \bar{\mu}_{1}=-0.021$, $\bar{\mu}_{2}=-0.013, \bar{\alpha}_{c}=\left[\begin{array}{lll}0.0078 & 0 & 0\end{array}\right]^{\prime}, \bar{\sigma}_{1}=\left[\begin{array}{lll}0 & 0.00034 & 0\end{array}\right], \bar{\sigma}_{2}=\left[\begin{array}{lll}0 & 0 & 0.038\end{array}\right]$.

\section{Incorporating jump risk}

So far, we have analyzed models formulated under Brownian information structures. In this section, we develop formulas that incorporate jumps in levels of the stochastic processes. We focus on a discrete state space specification with a finite number of states, where jumps are 

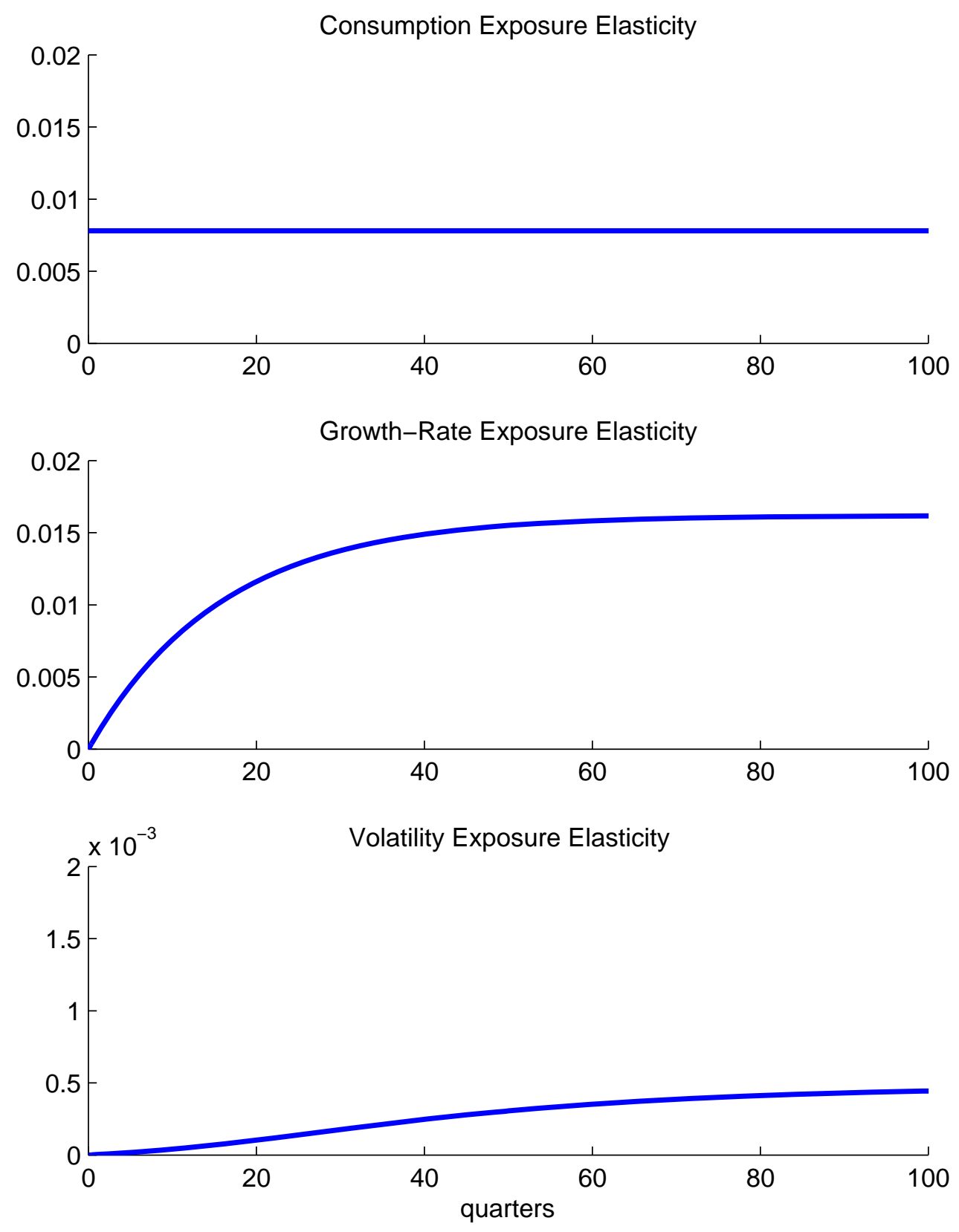

Figure 7: Shock-exposure elasticities for the aggregate consumption process parameterized as in Figure 6.

modeled as Poisson arrivals. 


\section{$7.1 \quad$ Basics}

Consider a functional $M$ of the form

$$
\log M_{t}=\sum_{0<u \leq t}\left(Z_{u-}\right)^{\prime} \kappa Z_{u}+\int_{0}^{t}\left(Z_{u-}\right)^{\prime} \beta d u+\int_{0}^{t}\left(Z_{u-}\right)^{\prime} \alpha d W_{u}
$$

Here $Z$ evolves as an $n$-state Markov chain with intensity matrix $A$ and the realizations of $Z$ are identified by a coordinate vector in $\mathbb{R}^{n}$. We write $Z_{t-}$ for the pre-jump (left) limit at date $t$. Abusing notation a bit, we now let $\beta$ be an $n$-dimensional vector and $\alpha$ an $n \times k$ matrix. The functional is now parameterized by the triplet $(\beta, \alpha, \kappa)$, representing the local mean conditional on no jumps, the local diffusive volatility and the jumps in the functional. In this specification, the local trend and volatility depend (linearly) on the Markov state.

In our calculations in this section we use the following notational conventions. dvec $\{\cdot\}$ applied to a square matrix returns a column vector with the entries given by the diagonal entries of the matrix, and $\operatorname{diag}\{\cdot\}$ applied to a vector produces a diagonal matrix from a vector by placing entries of the vector on the corresponding diagonal entries of the constructed matrix. The symbol $\times$ used in conjunction with two matrices forms a new matrix by performing multiplication entry by entry. $\exp ^{*}(\cdot)$ when applied to a vector or matrix performs exponentiation entry by entry. Finally, a real-valued function on the state space of coordinate vectors can be represented as a vector.

\subsubsection{Multiplicative martingales}

We construct a multiplicative martingale decomposition by computing an eigenfunction of the form $e \cdot z$ where the vector $e$ has all positive entries. The vector $e$ must solve the eigenvalue problem: ${ }^{13}$

$$
B e=\eta e
$$

where

$$
B \doteq \operatorname{diag}\left\{\beta+\frac{1}{2} \operatorname{dvec}\left\{\alpha \alpha^{\prime}\right\}\right\}+A \times \exp ^{*}(\kappa)
$$

Then

$$
M_{t}=\exp (\eta t) \hat{M}_{t}\left(\frac{e \cdot Z_{0}}{e \cdot Z_{t}}\right)
$$

and we can represent the martingale $\hat{M}$ as

$$
\log \hat{M}_{t}=\sum_{0<u \leq t}\left(Z_{u-}\right)^{\prime} \hat{\kappa} Z_{u}+\int_{0}^{t}\left(Z_{u-}\right)^{\prime} \beta d u+\int_{0}^{t}\left(Z_{u-}\right)^{\prime} \alpha d W_{u}-\eta t
$$

\footnotetext{
${ }^{13}$ Details on the construction of the eigenvalue problems can be found in Appendix B.1.
} 
where

$$
\hat{\kappa}=\kappa+\mathbf{1}_{n}(\log e)^{\prime}-(\log e) \mathbf{1}_{n}{ }^{\prime} .
$$

We use the multiplicative martingale $\hat{M}$ to change the probability measure. This measure change leads to a Brownian motion $\hat{W}$ under the new measure that satisfies

$$
d W_{t}=\left(Z_{t-}\right)^{\prime} \alpha d t+d \hat{W}_{t} .
$$

Under the new measure, the process $Z$ has intensity matrix

$$
\hat{A}=-\eta I+\operatorname{diag}(\hat{e}) B \operatorname{diag}(e)
$$

where $e$ and $\eta$ are given by the solution of the eigenvalue problem (31), and $\hat{e}$ is the vector of reciprocals of the entries in $e$.

\subsubsection{Additive martingales}

In order to construct perturbations corresponding to permanent shocks, we will extract the martingale component of an additive functional. Consider the martingale decomposition of the additive functional $\log M$ in (30)

$$
\log M_{t}=\rho t+\log \bar{M}_{t}-h \cdot Z_{t}+h \cdot Z_{0}
$$

To find the martingale component $\log \bar{M}$, let $q$ denote a vector with positive entries that sum to one and satisfy

$$
q^{\prime} A=0
$$

The long-run growth trend of the process is then given by

$$
\rho=q^{\prime} \operatorname{dvec}\left\{\kappa A^{\prime}\right\}+q^{\prime} \beta
$$

The vector $h$ determining the transient component can be found as the solution to

$$
A h=-\operatorname{dvec}\left\{\kappa A^{\prime}\right\}-\beta+\mathbf{1}_{n} \rho .
$$

Notice that the vector on the right-hand side is orthogonal to $q$, which is consistent with the fact that vectors in the image of $A$ are orthogonal to $q$ (see (36)). We solve equation (38) for $h$ restricting ourselves to the $n-1$ dimensional subspace of vectors that are orthogonal 
to $q$. The martingale component is then given by

$$
\log \bar{M}_{t}=\sum_{0<u \leq t}\left(Z_{u-}\right)^{\prime} \bar{\kappa} Z_{u}+\int_{0}^{t}\left(Z_{u-}\right)^{\prime} \bar{\beta} d u+\int_{0}^{t}\left(Z_{u-}\right)^{\prime} \alpha d W_{u}
$$

where

$$
\begin{aligned}
& \bar{\beta}=\beta-\mathbf{1}_{n} \rho \\
& \bar{\kappa}=\kappa+\mathbf{1}_{n} h^{\prime}-h \mathbf{1}_{n}^{\prime} .
\end{aligned}
$$

Observe that $\bar{\kappa}$ has again zeros on the main diagonal. The permanent component of the jump risk is thus given by

$$
\left(Z_{t-}\right)^{\prime} \bar{\kappa} Z_{t}+\left(Z_{t-}\right)^{\prime} \bar{\beta} d t
$$

We will also directly construct martingales. Consider an $n \times n$ matrix $\tilde{\kappa}$ with zeros on the diagonal, and build the additive martingale

$$
\log \tilde{M}_{t}=\sum_{0<u \leq t}\left(Z_{u-}\right)^{\prime} \tilde{\kappa} Z_{u}-\int_{0}^{t}\left(Z_{u-}\right)^{\prime} \operatorname{dvec}\left\{\tilde{\kappa} A^{\prime}\right\} d u
$$

For instance, we could specify all of the entries of $\tilde{\kappa}$ to be zero except for a single one.

Additive martingales scaled by the $\frac{1}{\sqrt{t}}$ obey the Central Limit Theorem. To deduce the variance $\varsigma^{2}$ associated with the normal approximation, the conditional second moment of the increment (per unit of time) is: ${ }^{14}$

$$
z^{\prime}(\tilde{\kappa} \times \tilde{\kappa}) A^{\prime} z
$$

Using the stationary distribution to average over alternative realizations of $z$,

$$
\varsigma^{2}=q \cdot \operatorname{dvec}\left\{(\tilde{\kappa} \times \tilde{\kappa}) A^{\prime}\right\}
$$

By scaling the matrix $\tilde{\kappa}$ by the scalar $\frac{1}{\varsigma}$, we obtain an additive martingale with a unit variance per unit of time.

\footnotetext{
${ }^{14}$ Locally the second moment and variance coincide.
} 


\subsubsection{Jump-risk perturbations}

Our jump-risk perturbations of a functional $M$ are of the form $M H(\mathrm{r})$ where

$$
\log H_{t}(\mathbf{r})=\sum_{0<u \leq t}\left(Z_{u-}\right)^{\prime}\left(\mathrm{r} \kappa_{d}\right) Z_{u}+\int_{0}^{t}\left(Z_{u-}\right)^{\prime} \beta_{h}(\mathrm{r}) d u
$$

where the direction matrix, $\kappa_{d}$, is the appropriately scaled (say $\frac{1}{\varsigma} \tilde{\kappa}$ ) jump risk component in the direction of the desired perturbation, and $\beta_{h}(r)$ is a vector that induces $H(r)$ or $G H(r)$ to be a martingale, depending on the application. For the former, $\beta_{h}(r)$ needs to satisfy

$$
0=\beta_{h}(\mathrm{r})+\operatorname{dvec}\left\{\exp ^{*}\left(\mathrm{r} \kappa_{d}\right) A^{\prime}\right\}
$$

Defining $\beta_{d}$ analogously to the diffusion case, we have

$$
\beta_{d}=\left.\frac{d \beta_{h}(r)}{d r}\right|_{r=0}=-\operatorname{dvec}\left\{\kappa_{d} A^{\prime}\right\}
$$

For the latter, recall that $G$ is parameterized by $\left(\beta_{g}, \alpha_{g}, \kappa_{g}\right)$. Since the coefficients are additive, the appropriate martingale restriction determining $\beta_{h}(r)$ is

$$
0=\frac{1}{2} \operatorname{dvec}\left\{\alpha \alpha^{\prime}\right\}+\beta+\beta_{h}(r)+\operatorname{dvec}\left\{\exp ^{*}\left(\kappa_{g}+\mathbf{r} \kappa_{\mathrm{d}}\right) A^{\prime}\right\}
$$

Differentiating with respect to $r$ and evaluating this derivative at zero, we have

$$
\beta_{d}=-\operatorname{dvec}\left\{\left[\exp ^{*}\left(\kappa_{g}\right) \times \kappa_{d}\right] A^{\prime}\right\}
$$

\subsubsection{Constructing the nonlinear moving-average representation}

We perform a direct calculation of the nonlinear moving-average coefficients needed for computing the elasticities that interest us. Recall that $\hat{e}$ is the vector of reciprocals of the entries of $e$. Then

$$
\begin{aligned}
\hat{e} \cdot Z_{t}= & \sum_{0<u \leq t}\left(Z_{u}-Z_{u-}\right)^{\prime} \exp [\hat{A}(t-u)] \hat{e}-\int_{0}^{t}\left(Z_{u-}\right)^{\prime} \hat{A} \exp [\hat{A}(t-u)] \hat{e} d u \\
& +\left(Z_{0}\right)^{\prime} \exp (\hat{A} t) \hat{e}
\end{aligned}
$$

where $\hat{A}$ is the intensity matrix under the change in measure. The new information at time $u$ is

$$
\left(Z_{u}-Z_{u-}\right)^{\prime} \exp [\hat{A}(t-u)] \hat{e}-\left(Z_{u-}\right)^{\prime} \hat{A} \exp [\hat{A}(t-u)] \hat{e} d u
$$


and the first two terms in the decomposition (42) form a martingale.

Next we scale by $\zeta(t-u)^{\prime} Z_{u-}$ where

$$
\zeta(t-u)=\exp [\hat{A}(t-u)] \hat{e}
$$

and produce a new representation that will be useful in our elasticity calculations. We do this in two steps:

1. First construct the matrix $\Xi(t-u)$ such that

$$
z^{\prime}[\Xi(t-u)] z^{*}=\frac{\zeta(t-u)^{\prime} z^{*}}{\zeta(t-u)^{\prime} z}-1
$$

2. Second construct the vector $\xi(t-u)$ by dividing each entry of $\hat{A} \zeta(t-u)$ by the corresponding entry of $\zeta(t-u)$.

Then write

$$
\begin{aligned}
\hat{e} \cdot Z_{t}=\sum_{0<u \leq t} & {\left[\zeta(t-u)^{\prime} Z_{u-}\right]\left[\left(Z_{u-}\right)^{\prime} \Xi(t-u) Z_{u}\right]-} \\
& -\int_{0}^{t}\left[\zeta(t-u)^{\prime} Z_{u-}\right]\left[\xi(t-u)^{\prime} Z_{u-}\right] d u+\zeta(t)^{\prime} Z_{0} .
\end{aligned}
$$

\subsubsection{Shock-price elasticities}

The moving-average representation for $\hat{e} \cdot Z_{t}$ derived in Section 7.1 .4 allows us to state a counterpart of Proposition 5.1 for the jump risk case.

Proposition 7.1. Let $M$ be a multiplicative functional parameterized by $(\beta, \alpha, \kappa)$ with martingale decomposition outlined in Section 7.1.1, $H(\mathrm{r})$ a jump perturbation of the form (41) parameterized by $\left(\beta_{h}(\mathrm{r}), 0, \mathrm{r} \kappa_{d}\right)$, and $\beta_{d}=d \beta_{h}(\mathrm{r}) /\left.d \mathbf{r}\right|_{\mathrm{r}=0}$. Then

$$
\begin{aligned}
& \left.\frac{d}{d \mathbf{r}} \log E\left[M_{t} H_{t}(\mathrm{r}) \mid Z_{0}=z\right]\right|_{\mathrm{r}=0}= \\
& \quad=\frac{\hat{E}\left[\left(\hat{e} \cdot Z_{t}\right) \int_{0}^{t}\left(Z_{u-}\right)^{\prime}\left(\beta_{d}+\operatorname{dvec}\left\{\kappa_{d} \hat{A}^{\prime}\right\}+\operatorname{dvec}\left\{\left(\Xi(t-u) \times \kappa_{d}\right) \hat{A}^{\prime}\right\}\right) d u \mid Z_{0}=z\right]}{\hat{E}\left[\hat{e} \cdot Z_{t} \mid Z_{0}=z\right]}
\end{aligned}
$$

The proof is deferred to Appendix B.2. Switching the order of integration in the numerator of formula (45), the shock elasticity function for a direction $\kappa_{d}$ is the time $u=0$ contribution to the integral across the time dimension, viewed as a function of the maturity date $t$. As in the Brownian case, we obtain the shock-price elasticity function in the special 
case when $M=S G$ and both $G$ and $G H(\mathrm{r})$ are martingales, attaching a minus sign by signing convention. We write the shock-price elasticity function as a vector

$$
\pi(t)=-\beta_{d}-\operatorname{dvec}\left\{\kappa_{d} \hat{A}^{\prime}\right\}-\operatorname{dvec}\left\{\left[\Xi(t) \times \kappa_{d}\right] \hat{A}^{\prime}\right\}
$$

\subsection{Growth and discounting}

Our construction of the multiplicative functional $M$ in (30) that explicitly allows for jumps in the levels of the functional is motivated by the implications of continuous-time Markov switching models for the dynamics of equilibrium quantities that are of interest to us. For the sake of illustration we introduce jumps directly in the growth or consumption processes, but production economies are also of interest and can be, and in fact have been, investigated using computational methods.

There are also other potential sources of jumps. We have already shown that even if a stochastic growth or discount functional contains no jumps in the sample paths $(\kappa=0)$, its martingale component both in additive and multiplicative form will generically contain a jump component provided there are jumps in either the conditional mean of the growth or conditional volatility. As we will see, when consumers have EZ preferences the forwardlooking continuation values may exhibit jumps even if the consumption process has a continuous sample path. ${ }^{15}$ Jumps thus become relevant when pricing permanent components of asset payoffs.

In some models with production and capital accumulation, jumps in the equilibrium consumption process may arise endogenously. In the Cagetti et al. (2002) model, the discrete Markov state determines the mean growth rate of the technology process, but the process itself has continuous trajectories. Since a regime shift discretely changes the instantaneous mean growth rate of the technology process and the conditional distribution of the future technology, there is also a discrete adjustment in the consumption and investment processes. $^{16}$

In the following subsections, we construct the stochastic discount factor functional for the continuous-time version of the Epstein and Zin (1989) preferences when intertemporal elasticity of substitution is equal to one. We will subsequently use the stochastic discount factor to calculate the shock-price elasticities for consumption dynamics estimated by Bonomo and

\footnotetext{
${ }^{15}$ Calvet and Fisher (2008), Chen (2008), and Bhamra et al. (2008) generate stochastic discount factors with discontinuous trajectories using the continuous-time version of Epstein and Zin (1989) preferences.

${ }^{16}$ David (1997) produces a model along similar lines with two different linear technologies where jumps in the mean growth rates of the two technologies exactly offset each other, so that the distribution of the aggregate production possibility set is independent of the current state. In this case, the equilibrium consumption process remains continuous.
} 


\begin{tabular}{rc|rrr}
\hline \hline$\beta_{c}$ & $\alpha_{c}$ & \multicolumn{3}{|c}{$A$} \\
\hline 0.0355 & 0.0330 & -0.4627 & 0.4627 & 0 \\
0.0127 & 0.0484 & 0.1709 & -0.1938 & 0.0229 \\
0.0193 & 0.0163 & 0.0554 & 0 & -0.0554 \\
\hline \hline
\end{tabular}

Table 1: Parameterization of the jump risk example, annualized quantities. The intensity matrix is calculated by taking the matrix logarithm of the transition probability matrix from Bonomo and Garcia (1996), and setting all negative off-diagonal terms equal to zero. This produces an intensity matrix with zeros in the same entries as in the original transition probability matrix. Original parameters estimated using yearly data from 1889-1985 (for details on the data sources, see Appendix A of the cited paper).

Garcia (1996).

\subsection{Example economy with jumps}

\subsubsection{State dynamics}

For illustrative purposes, we consider an example of consumption dynamics with three states, estimated by Bonomo and Garcia (1996). We focus on the pricing of permanent jump shocks to the equilibrium consumption stream in the Breeden-Lucas (BL) and Epstein-Zin (EZ) specification of preferences.

Bonomo and Garcia (1996) specify the consumption dynamics as a conditionally Gaussian discrete-time process with jumps in the conditional growth rate and volatility. This leads us to parameterize consumption as a multiplicative functional given by $\left(\beta_{c}, \alpha_{c}, \kappa_{c}\right)$ where $\kappa_{c}=0$ and scaled by the initial condition $C_{0}$. Table 1 provides the parameter values $\beta_{c}$ and $\alpha_{c}$ estimated by Bonomo and Garcia (1996), while Figure 8 gives the filtered probabilities of the three states. Before 1950, the economy was mostly switching between states 1 and 2, with longer spells spent in state 2. These were the more volatile times in the first half of the $20^{\text {th }}$ century. After 1950, the economy switches to the highly persistent, low-volatility, average-mean growth rate state 3 , where it resides for most of the remainder of the sample (at least until recent events).

\subsubsection{Investors' preferences}

As in Section 6.2 we consider two models of preferences. Recall that in model BL the stochastic discount functional is:

$$
S_{t}=\exp (-\delta t)\left(\frac{C_{t}}{C_{0}}\right)^{-\gamma} .
$$




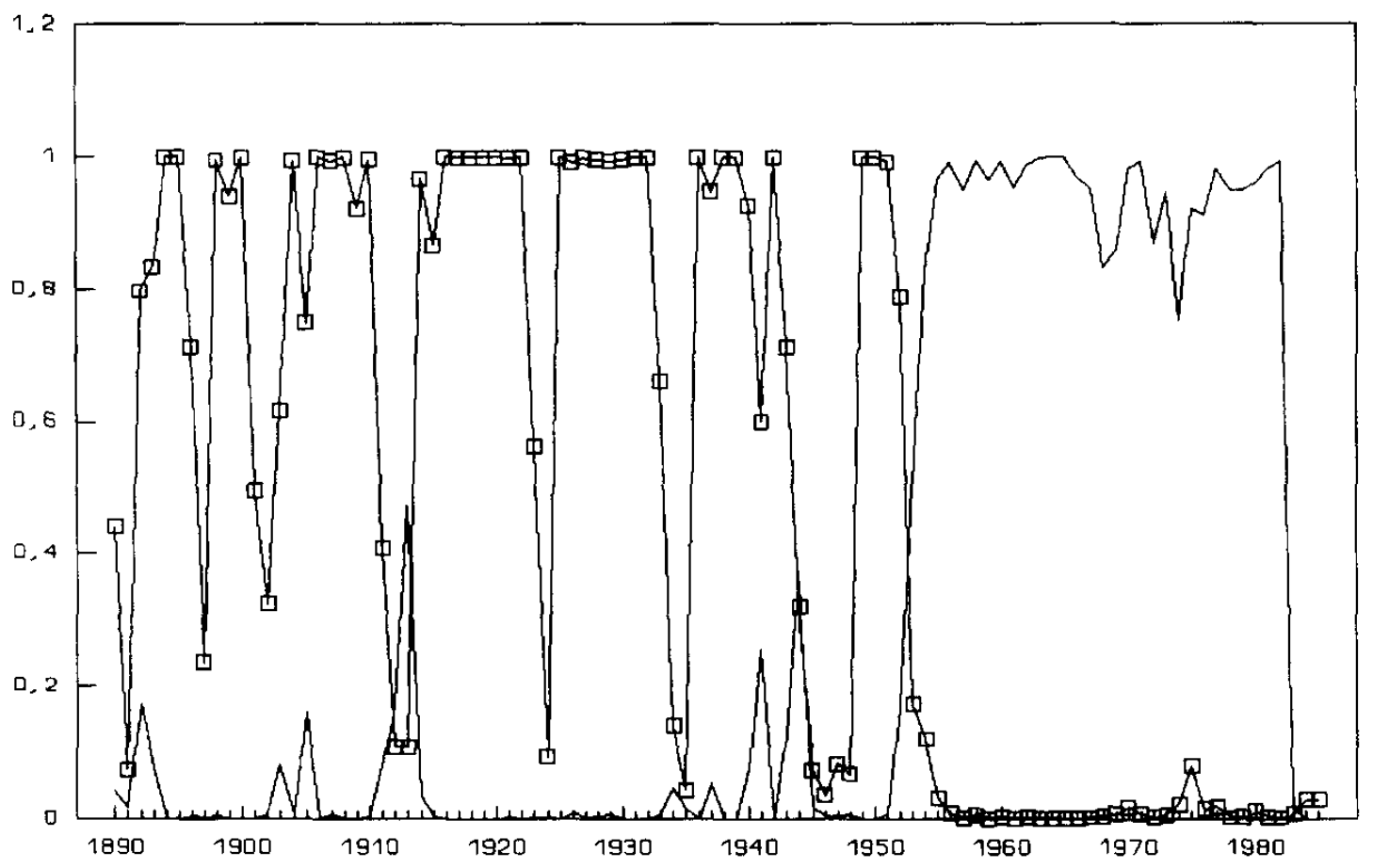

Figure 8: This is Figure 3 from Bonomo and Garcia (1996). The curve with boxes depicts the filtered probabilities for state two and the solid curve without boxes depicts the filtered probabilities for state three.

In model EZ we use the continuous-time specification of recursive utility preferences given in Section 6.2. As we saw, when preferences have a unitary elasticity the stochastic discount functional has a particularly simple form:

$$
S_{t}=\exp (-\delta t)\left(\frac{C_{0}}{C_{t}}\right) \tilde{S}_{t}
$$

where $\tilde{S}_{t}$ is the multiplicative martingale component of

$$
\left(\frac{V_{t}}{V_{0}}\right)^{1-\gamma}
$$

As in Hansen (2007), the continuation value is of the form

$$
\log V_{t}=v \cdot Z_{t}+\log C_{t}
$$

where $v$ solves the continuous-time discrete-state Bellman equation:

$$
0=-\delta v+\beta_{c}+\frac{1}{1-\gamma} \operatorname{dvec}\left\{\exp ^{*}\left[(1-\gamma) \kappa_{v}\right] A^{\prime}\right\}+\frac{1-\gamma}{2} \operatorname{dvec}\left\{\alpha_{c} \alpha_{c}^{\prime}\right\}
$$


and ${ }^{17}$

$$
\kappa_{v}=\left(\mathbf{1}_{n} v^{\prime}-v \mathbf{1}_{n}{ }^{\prime}\right)+\kappa_{c}
$$

To construct this martingale component, write

$$
\begin{aligned}
(1-\gamma)\left(\log V_{t}-\log V_{0}\right) & =(1-\gamma)\left[v \cdot\left(Z_{t}-Z_{0}\right)+\log C_{t}-\log C_{0}\right] \\
& =(1-\gamma)\left[\sum_{0<u \leq t} Z_{u-}{ }^{\prime} \kappa_{v} Z_{u}+\int_{0}^{t} Z_{u-}{ }^{\prime} \beta_{c} d u+\int_{0}^{t} Z_{u-}{ }^{\prime} \alpha_{c} d W_{u}\right]
\end{aligned}
$$

Then

$$
\begin{aligned}
\log \tilde{S}_{t}= & (1-\gamma) \sum_{0<u \leq t} Z_{u-}{ }^{\prime} \kappa_{v} Z_{u}-\int_{0}^{t} \operatorname{dvec}\left\{\exp ^{*}\left[(1-\gamma) \kappa_{v}\right] A^{\prime}\right\} \cdot Z_{u-} d u \\
& +(1-\gamma) \int_{0}^{t} Z_{u-}{ }^{\prime} \alpha_{c} d W_{u}-\int_{0}^{t} \operatorname{dvec}\left\{\frac{(1-\gamma)^{2}}{2} \alpha_{c} \alpha_{c}{ }^{\prime}\right\} \cdot Z_{u-} d u
\end{aligned}
$$

The coefficients in the stochastic discount functional thus are

$$
\begin{aligned}
\beta_{s} & =-\delta \mathbf{1}_{n}-\beta_{c}-\operatorname{dvec}\left\{\exp ^{*}\left[(1-\gamma) \kappa_{v}\right] A^{\prime}\right\}-\operatorname{dvec}\left\{\frac{(1-\gamma)^{2}}{2} \alpha_{c} \alpha_{c}{ }^{\prime}\right\} \\
& =-\delta \mathbf{1}_{n}-(1-\gamma) \delta v-\gamma \beta_{c} \\
\alpha_{s} & =-\alpha_{c}+(1-\gamma) \alpha_{c}=-\gamma \alpha_{c} \\
\kappa_{s} & =-\kappa_{c}+(1-\gamma) \kappa_{v}=-\gamma \kappa_{c}+(1-\gamma)\left(\mathbf{1}_{n} v^{\prime}-v \mathbf{1}_{n}{ }^{\prime}\right) .
\end{aligned}
$$

where we have used equation (48) for the vector $\delta v$.

\subsubsection{Elasticities and risk-price increments}

We specify the growth functional $G$ as the multiplicative martingale component of the consumption functional $C$, extracted using the procedure outlined in Section 7.1.1. The first perturbation we consider is the jump component of the permanent shock to $\log C$. Let $\bar{\kappa}_{c}$ denote the corresponding jump matrix, dictating how the shock is constructed as function of the jumps in $Z$. We parameterize the perturbation $H(r)$ using $\left(\beta_{h}(r), 0, r \bar{\kappa}_{\mathrm{c}}\right)$ where $\beta_{h}(r)$ makes $G H(r)$ a martingale, and then we scale the perturbation by the reciprocal of the longrun volatility as in Section 7.1.2. This scaling normalizes the risk exposure of the shock.

\footnotetext{
${ }^{17}$ This equation is more general than the corresponding equation in Hansen (2007) because it allows for jumps in the consumption process and heteroskedasticity in the loading on the Brownian increment.
} 

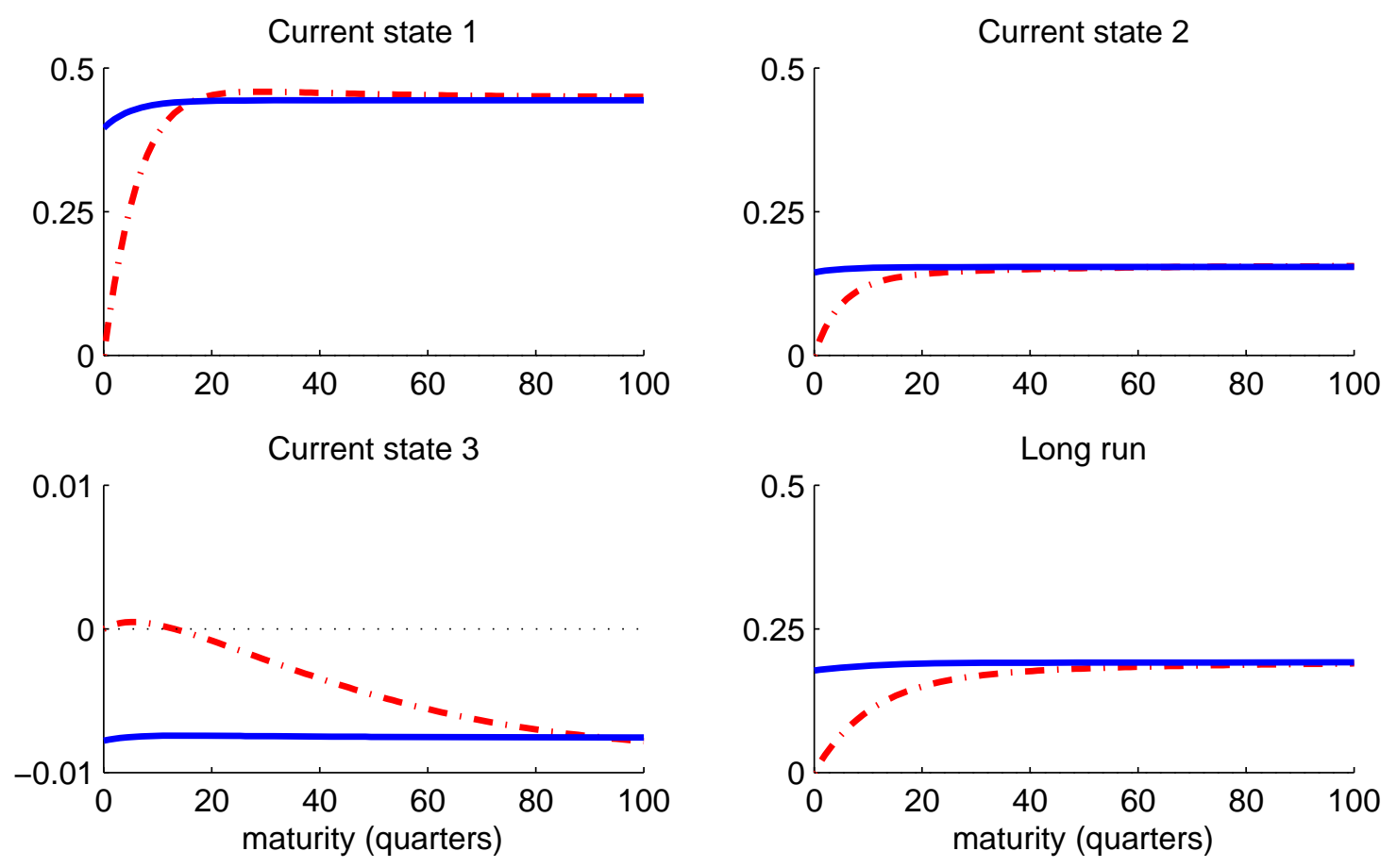

Figure 9: Shock-price elasticities for the Bonomo and Garcia (1996) consumption dynamics under the Breeden-Lucas (dot-dashed lines) and Epstein-Zin (solid lines) utility specifications. The priced growth functional is the martingale component in the multiplicative decomposition of $C$, and the direction of the perturbation is given by the jump component of the martingale in the additive decomposition of $\log C$. Preference parameters are $\gamma=10$ and $\delta=0.01$.

The resulting direction matrix is:

$$
\frac{1}{\varsigma} \bar{\kappa}_{c}=\left(\begin{array}{rrr}
0 & -2.1804 & -0.1139 \\
2.1804 & 0 & 2.0664 \\
0.1139 & -2.0664 & 0
\end{array}\right)
$$

As reflected by the first row of this matrix, a movement from the first state to either of the other states had an adverse consequence on this permanent shock to consumption. In contrast, movements from the second state to either of the first two states has a positive impact on the permanent shock. From the third state, a movement to the high growth first state has a positive impact and to the low growth second state a negative impact.

The shock-price elasticities (46) for the two utility specifications are shown in Figure 9. We plot the elasticities conditional on each of the three states, as well as the limiting contribution (19).

In the BL model the paths of the stochastic discount factor $S$ are continuous. Since 
Current state 1

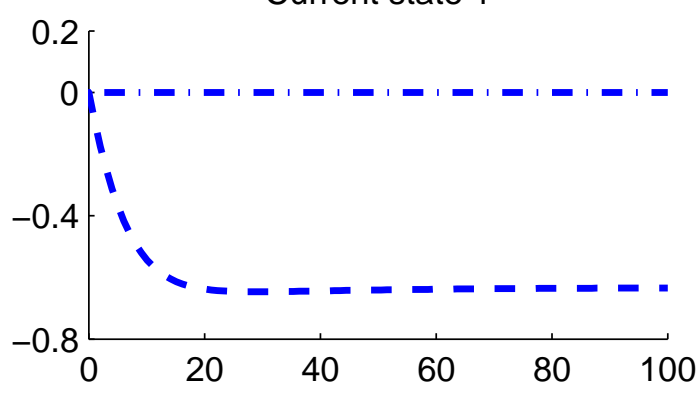

Current state 3

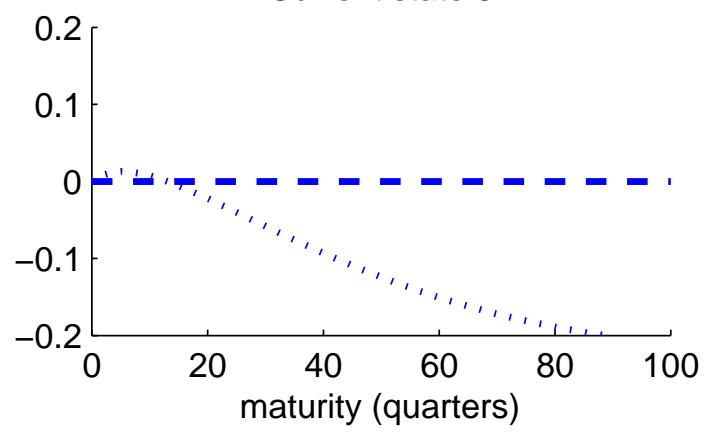

Current state 2
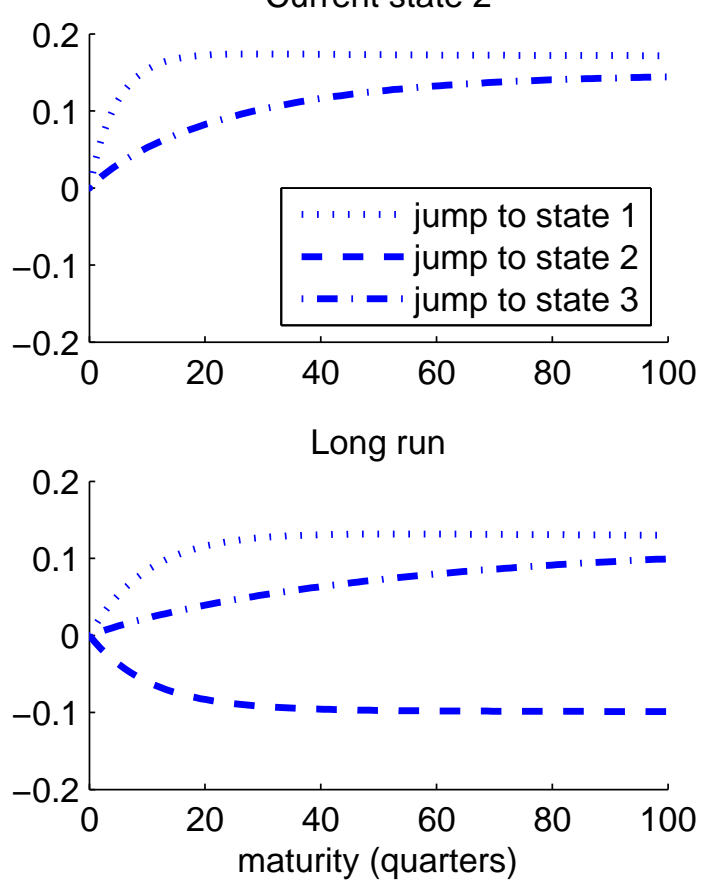

Figure 10: Shock-price elasticities for the Bonomo and Garcia (1996) consumption dynamics for the BL specification. The growth functional $G$ is the martingale component in the multiplicative decomposition of $C$. We set the direction matrices to feature each of the three states, adjust the local mean to make $G H(r)$ a martingale, and scale by the reciprocal of the long-run volatility. The preference parameters are $\gamma=10$ and $\delta=0.01$.

the diffusion and jump terms are constructed as having independent increments, the local elasticity with respect to the jump component is zero. However, as we have already indicated, our construction of the growth functional $G$ as the martingale component of $C$ includes a nonzero jump part. The elasticity functions flatten out for maturities over 5 years. In the EZ specification, the elasticity trajectories are almost flat, reflecting the dominance in the martingale component of the stochastic discount factor.

From Figure 9, we see that the elasticity functions flatten out for maturities over 5 years. In the EZ specification, the elasticity trajectories are almost flat, reflecting the dominance in the martingale component of the stochastic discount factor. Also the price of the immediate exposure to permanent jump shock varies substantially depending upon the current state. While the state one prices are sizeable, the state three prices are tiny. Recall that filtered state probabilities indicate that the economy was switching between states 1 and 2 until about 1950, while in the post-1950 era the economy mostly resided in state 3 . The limiting shock-price trajectory, (19), is plotted in the bottom right panel of Figure 9. This curve shows that a forward shift in the time of exposure in the case of state three will substantially 
increase the risk prices while the same shift in case of state one leads to a drop by about fifty percent.

We explore the pricing of exposure to each of the states for the BL specification of preferences. We construct direction matrices $\kappa_{d}$ to feature jumps into the three states. For each state we start with a direction matrix of zeros except in the column corresponding to the state of interest say jump state $j$. Column $j$ has ones in all entries except for the diagonal entry. We again divide by the long-run volatility, and we make the appropriate drift compensations so that $G H(r)$ is a martingale. The resulting shock-price elasticities are shown in Figure 10. Each of three panels corresponds to a different current state. For each current state we depict the shock-price elasticities for jumping into the other states. Some of these curves are degenerate because it is not possible to jump immediately from state 3 to state 2 and from state 1 to state 3 . (See Table 1.)

The speed of convergence of the nondegenerate elasticity functions is dictated by the implied jump intensities. Recall that state 2 is the low-growth, high volatility state. Exposure to an upward jump from state 1 to state 2 is desirable and purchasing a claim on such an outcome provides insurance. As a consequence the shock-price elasticity function is negative. Exposure to transition from state 3 to 1 is also associated with a negative shockprice elasticity for long-horizon cash flows. This reflects in part the indirect effect that if state 1 is realized this increases the possibility that low growth-high volatility state 2 will be reached prior to the date of the payoff. We also include the limiting shock price trajectories in the bottom-right panel. In the long-term the jump exposure to state 1 exceeds that of state 3 . The exposure to state 2 commands a negative shock price, again because of the insurance motive.

The constructed shock-price elasticities represent the sensitivity of values of cash flows to a shock over the next instant. We now consider the impact of shocks at intermediate dates between now and the maturity of the cash flow. Recall that we termed these intermediate contributions risk-price increments because they effectively integrate to risk price (elasticity) over the payoff horizon. (See Proposition 7.1.) As in the continuous-state case, we calculate the risk-price increments by taking conditional expectations of the shock-price elasticities at intermediate dates using distorted probability distributions. This calculation combines the state dependence of the elasticity function with the impact of stochastic growth and discounting in the conditional expectation. Formula (45) implies a time $u, 0 \leq u \leq t$, contribution

$$
\begin{aligned}
-(\operatorname{diag}\{\zeta(t)\})^{-1} \exp (\hat{A} u)[\zeta(t-u) \times \\
\left.\times\left(\beta_{d}+\operatorname{dvec}\left\{\kappa_{d} \hat{A}^{\prime}\right\}+\operatorname{dvec}\left\{\left[\Xi(t-u) \times \kappa_{d}\right] \hat{A}^{\prime}\right\}\right)\right]
\end{aligned}
$$



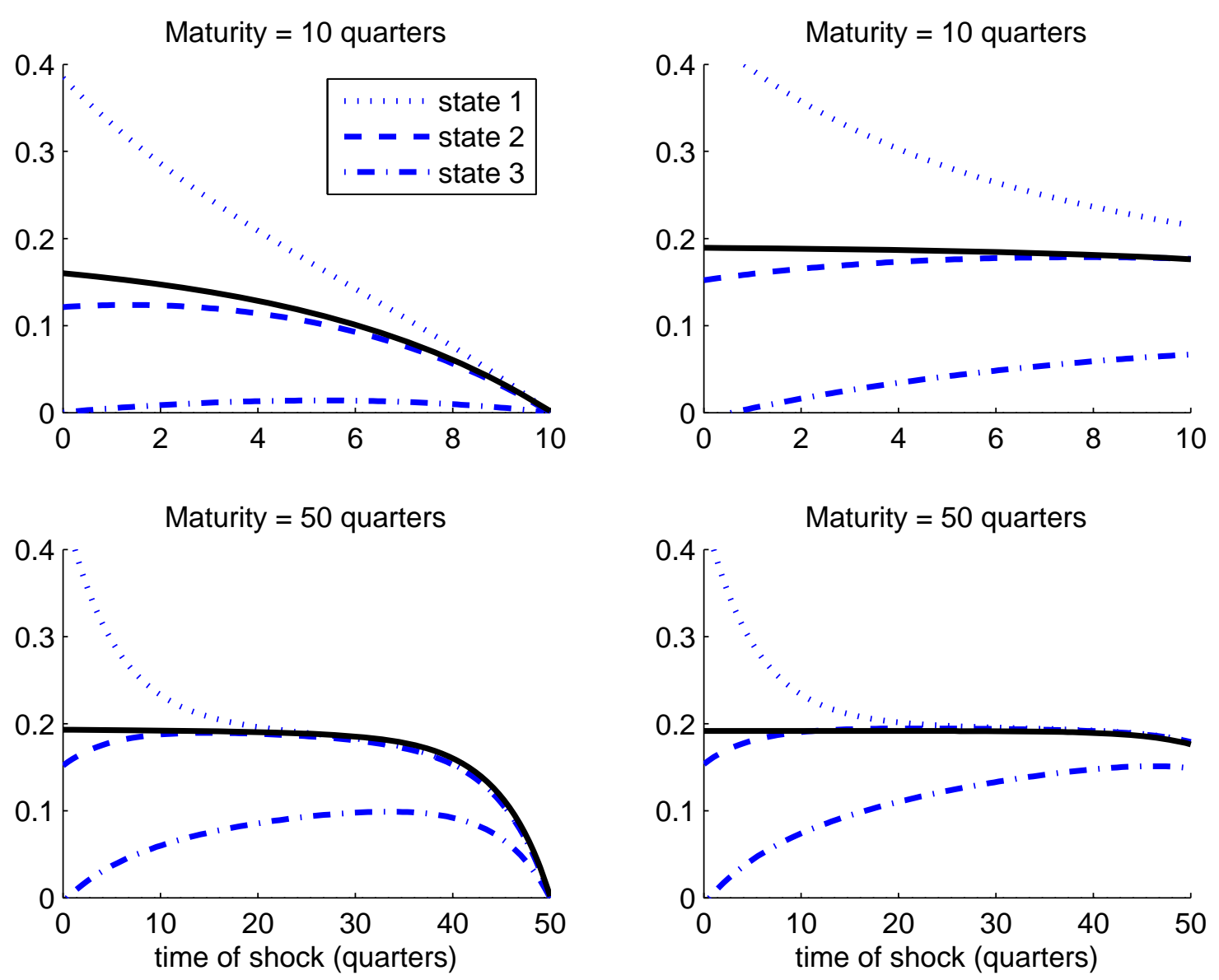

Figure 11: Risk-price increments for the Bonomo and Garcia (1996) consumption dynamics for the BL (left column) and EZ (right column) preference specifications. Individual panels depict the risk-price increments for payoffs of different maturities. The solid black line corresponds to the long-run elasticity curve from Figure 9, plotted backwards from the maturity date. Risk prices are obtained by integrating under the individual curves and scaling by $1 / t$. The preference parameters are $\gamma=10$ and $\delta=0.01$.

We depict these risk-price increments for the BL and EZ models in Figure 11 for exposure to the jump component of the permanent shock to consumption constructed previously. Each panel represents the decomposition of the risk price for the cash flow of a given maturity into the contributions of shocks at different horizons.

We first focus on the risk-price increments for the BL model shown in the left column of Figure 11. The plots capture substantial state dependence in risk-price increments for proximate shocks, and their convergence as the time of the shocks moves further to the future. Consistent with the plot for the shock-price elasticities, the risk-price increments in model BL diminish to zero as the time of shock approaches the maturity date. Notice that in state three, the state commonly visited during the post war, the risk-price increments remain 
small for an investment horizon of ten quarters but the increments are more notable for the fifty-quarter horizon as the probability of transiting to a bad state becomes less negligible. Thus in state three the risk prices are more substantial over the longer time horizons in the BL model.

Next we compare the risk-price increments in the BL model to those in the EZ model. The EZ model risk-price increments are displayed in the right column of Figure 11. The important differences are in the risk-price increments that are close to the maturity date. In the EZ model there is no drop off in the price increments near the terminal date of the payoff. For the ten quarter maturity the overall difference between models remains substantial. As the payoff date is extended to fifty quarters the risk-price increments behave similarly across models for the first forty quarters (ten years) and then the BL risk-price increments diminish to zero over the remaining quarters of the investment horizon. Shockprice elasticities and their counterparts for shocks at intermediate dates thus reveal pricing subtleties across models that are disguised by the prices of risk exposures over the entire investment horizon.

\section{Conclusion}

Stochastic dynamic model economies inform us how alternative shocks influence key economic variables at alternative time horizons. Structural models of asset valuation tell us even more. They inform us how the exposure to nondiversifiable macroeconomic shocks is compensated over alternative investment horizons. To understand better such implications, we proposed shock-price elasticities that measure this compensation and are valuation counterparts to impulse response functions. These price elasticities are also the dynamic extension of local risk prices familiar from finance. Similarly, we constructed shock-exposure elasticities which capture the sensitivity of expected cash flows. We produced tractable continuous-time formulas for structural models that explicitly account for stochastic discounting and macroeconomic growth. Thus this paper provides an additional tool for analyzing structural models that connect macroeconomics and asset pricing.

In this paper we deduced price and exposure elasticities by deconstructing the risk premia of conveniently chosen cash flows. Risk premia on specific assets depend on the exposure of an underlying cash flow to risk along with the price of that exposure. By design our elasticity calculations explore marginal changes in exposures in alternative directions, and in models with nonlinearities these elasticities depend on what benchmark cash flow is used in their construction and on the evolution of the Markov state. Constructing risk premia thus requires that we integrate the marginal contributions over the range of the relevant 
exposures. This integration is implicit when we confront empirical evidence using a limited set of asset payoffs and prices. While we have not proposed a new set of statistical procedures for testing, we believe the deconstruction of risk premia to be of interest in understanding better the implications of alternative asset pricing models.

In a series of examples, we showed how to construct the shock elasticities in models where investors' preferences include external habit or recursive utility, and where there is consumption predictability and stochastic volatility, so called "long-run risks." We also explored models where the dynamics are driven, at least in part, by a finite-state Markov chain. We showed examples in which models that have similar implications for local risk prices have dramatically different implications over long investment horizons, and examples of models that have very similar long-term price implications but substantially different implications for shorter horizons.

While our examples feature alternative specifications of investor preferences, the starting point for the methods we develop is a benchmark macroeconomic growth process and a corresponding stochastic discount factor process. It is well known that models with explicit investor heterogeneity in opportunities and limitations to the nature of asset trading can still be captured by appropriately specified stochastic discount factors. For instance, see Hansen and Renault (2009). We anticipate that a more comprehensive study of the pricing implications of these models will reveal interesting comparisons to some of the models that we have explored in this paper.

Finally, we have abstracted from econometric and empirical challenges. While we leave this to future work, we do not wish to diminish the importance of these tasks. In regard to empirical implications, Bansal and Lehmann (1997), Alvarez and Jermann (2005), and Koijen et al. (2009) use the holding period return on long-term bonds and the maximal growth portfolio to gain information about the one-period stochastic discount factor in a discrete-time asset pricing model. The risk premium on the maximal growth portfolio reveals information on the volatility of the logarithm of the stochastic discount factor and the limiting holding-period return on a discount bond reveals the one-period ratio of the dominant eigenfunction $\left(e\left(X_{t+1}\right) / e\left(X_{t}\right)\right.$ in our notation) in a multiplicative factorization of the stochastic discount factor. Taken together, they construct informative bounds on the logarithm of the stochastic discount factor and its components. The specification of stochastic growth risk exposure plays no role in their analyses, and thus empirical extensions of this literature that explicitly confront the valuation of stochastic growth is a potentially fruitful direction for future research. The initial steps by Lettau and Wachter (2007) and Hansen et al. (2008) are promising starts in this direction. 


\section{A Derivations for Section 6.2}

Consider recursive preferences in the case of unitary elasticity of intertemporal substitution. Hansen (2009) shows that when the subjective rate of time preference $\delta \searrow 0$, the stochastic discount factor is of the following form:

$$
S_{t}^{*}=\exp (-\eta t) S_{t} \frac{e\left(X_{t}\right)}{e\left(X_{0}\right)}
$$

where $S$ is the Breeden-Lucas stochastic discount factor specified in Section 6.2, and $\eta$ and $e(x)=\exp \left(\lambda^{\prime} x\right)$ are the eigenvalue and eigenfunction associated with the principal martingale decomposition of $C^{1-\gamma}$.

In order to find the eigenvalue and eigenfunction for a general multiplicative functional $M$ of form (26), decompose $\lambda$ into a vector $\lambda_{1}$ and its final entry, $\lambda_{2}$. Then $\lambda$ solves the pair of equations

$$
\begin{aligned}
& 0=\bar{\mu}_{1}^{\prime} \lambda_{1}+\bar{\beta}_{1}^{\prime} \\
& 0=\bar{\mu}_{2} \lambda_{2}+\bar{\beta}_{2}+\frac{1}{2}\left|\lambda_{1}^{\prime} \bar{\sigma}_{1}+\lambda_{2} \bar{\sigma}_{2}+\bar{\alpha}\right|^{2}
\end{aligned}
$$

Additionally, the associated eigenvalue is given by

$$
\eta=\bar{\beta}_{0}-\left(\bar{\mu}_{2} \lambda_{2}+\bar{\beta}_{2}\right)
$$

Since equation (51) has in general multiple solutions, we follow Hansen and Scheinkman (2009a) and choose the solution that is associated with the smallest eigenvalue. This solution is the one that leads to stable dynamics of the Markov process $X$ under the change of measure.

It follows from the specification of $e(x)$ that the stochastic discount factor $S^{*}$ is also of form (26). The coefficients are given by

$$
\begin{aligned}
\bar{\beta}_{s, 0}^{*} & =-\delta-\bar{\beta}_{c, 0}+(1-\gamma) \bar{\beta}_{c, 2}+\lambda_{2} \bar{\mu}_{2} \\
\bar{\beta}_{s, 1}^{*} & =-\gamma \bar{\beta}_{c, 1}+\lambda_{1}^{\prime} \bar{\mu}_{1} \\
\bar{\beta}_{s, 2}^{*} & =-\gamma \bar{\beta}_{c, 2}+\lambda_{2} \bar{\mu}_{2} \\
\bar{\alpha}_{s}^{*} & =-\gamma \bar{\alpha}_{c}+\lambda_{1}^{\prime} \bar{\sigma}_{1}+\lambda_{2} \bar{\sigma}_{2}
\end{aligned}
$$

Finally, Hansen (2009) shows that for a multiplicative functional $M$ parameterized by $(26)$

$$
E\left[M_{t} \mid X_{0}=x\right]=\exp \left\{\theta_{0}(t)+\theta_{1}(t) \cdot x^{[1]}+\theta_{2}(t) x^{[2]}\right\}
$$


where the $\theta_{i}(t)$ coefficients satisfy the following set of ordinary differential equations, each with initial condition $\theta_{i}(0)=0$.

$$
\begin{aligned}
\frac{d}{d t} \theta_{1}(t) & =\bar{\beta}_{1}^{\prime}+\bar{\mu}_{1}^{\prime} \theta_{1}(t) \\
\frac{d}{d t} \theta_{2}(t) & =\bar{\beta}_{2}+\bar{\mu}_{2} \theta_{2}(t)+\frac{1}{2}\left|\bar{\alpha}+\theta_{1}(t)^{\prime} \bar{\sigma}_{1}+\theta_{2}(t) \bar{\sigma}_{2}\right|^{2} \\
\frac{d}{d t} \theta_{0}(t) & =\bar{\beta}_{0}-\bar{\beta}_{2}-\bar{\mu}_{2} \theta_{2}(t)
\end{aligned}
$$

\section{B Derivations and proofs for Section 7}

\section{B.1 Eigenvalue problems}

For the multiplicative decomposition in Section 7.1.1, guess that the martingale component takes the form (33). The martingale restriction for an increment in $\hat{M}$ conditional on state $Z_{t-}=z_{i}$ is

$$
0=\frac{1}{2} z_{i}^{\prime} \alpha \alpha^{\prime} z_{i}+\beta_{i}-\eta+\sum_{j} A_{i j} \exp \left(\hat{\kappa}_{i j}\right)
$$

Plugging this restriction into decomposition (32) and comparing coefficients, we obtain the condition

$$
\hat{\kappa}_{i j}=\log e_{j}-\log e_{i}+\kappa_{i j}
$$

which yields equation (34) by stacking. Using this condition in the martingale restriction leads to

$$
0=\frac{1}{2} z_{i}^{\prime} \alpha \alpha^{\prime} z_{i}+\beta_{i}-\eta+\sum_{j} A_{i j} \frac{e_{j}}{e_{i}} \exp \left(\kappa_{i j}\right)
$$

which, after multiplying by $e_{i}$ and stacking the equations, yields the eigenvalue equation (31).

The additive decomposition in Section 7.1.2 is obtained in a similar way. Guess the form of the martingale component $\log \bar{M}$ given by equation (39). The additive martingale restriction conditional on state $Z_{t-}=z_{i}$ is

$$
0=\sum_{j} \bar{\kappa}_{i j} A_{i j}+\bar{\beta}_{i}
$$

Using this restriction in (35), and comparing coefficients, we have

$$
\begin{aligned}
\bar{\beta}_{i} & =\beta_{i}-\rho \\
\bar{\kappa}_{i j} & =\kappa_{i j}+h_{j}-h_{i}
\end{aligned}
$$


Thus the martingale restriction implies

$$
\rho=\sum_{j} \kappa_{i j} A_{i j}+\sum_{j} A_{i j} h_{j}+\beta_{i}
$$

Stacking this set of equations and premultiplying by $q$ yields equation (37). The vector of transient components $h$ can then be found (up to scale) as a solution to the system of equations (52).

\section{B.2 Proof of Proposition 7.1}

Proof. Notice that

$$
\left.\frac{d}{d r} \log E\left[M_{t} H_{t}(\mathrm{r}) \mid Z_{0}=z\right]\right|_{\mathrm{r}=0}=\frac{\hat{E}\left[\left(\hat{e} \cdot Z_{t}\right) D_{t} \mid Z_{0}=z\right]}{\hat{E}\left[\hat{e} \cdot Z_{t} \mid Z_{0}=z\right]}
$$

where

$$
D_{t}=\sum_{0<u \leq t}\left(Z_{u-}\right)^{\prime} \kappa_{d} Z_{u}+\int_{0}^{t}\left(Z_{u-}\right)^{\prime} \beta_{d} d u .
$$

Notice that the additive functional

$$
\bar{D}_{t}=\sum_{0<u \leq t}\left(Z_{u-}\right)^{\prime} \kappa_{d} Z_{u}-\int_{0}^{t}\left(Z_{u-}\right)^{\prime} \operatorname{dvec}\left\{\kappa_{d} \hat{A}^{\prime}\right\} d u
$$

is a martingale under the change of probability measure. In order to find the expression for $\hat{E}\left[\left(\hat{e} \cdot Z_{t}\right) \bar{D}_{t} \mid Z_{0}=z\right]$, we calculate the local covariance between corresponding increments in $\bar{D}$ and the moving-average decomposition of $\hat{e} \cdot Z_{t}$ in formula (43). We have

$$
\begin{aligned}
& \hat{E}\left[\left(\hat{e} \cdot Z_{t}\right) \bar{D}_{t} \mid Z_{0}=z\right]= \\
& \quad=\hat{E}\left[\int_{0}^{t}\left[\zeta(t-u)^{\prime} Z_{u-}\right]\left[\left(Z_{u-}\right)^{\prime} \operatorname{dvec}\left\{\left(\Xi(t-u) \times \kappa_{d}\right) \hat{A}^{\prime}\right\}\right] d u \mid Z_{0}=z\right] \\
& \quad=\hat{E}\left[\int_{0}^{t}\left(\hat{e} \cdot Z_{t}\right)\left[\left(Z_{u-}\right)^{\prime} \operatorname{dvec}\left\{\left(\Xi(t-u) \times \kappa_{d}\right) \hat{A}^{\prime}\right\}\right] d u \mid Z_{0}=z\right]
\end{aligned}
$$

where we used

$$
z^{\prime}\left[\left(\Xi(t-u) \times \kappa_{d}\right) \hat{A}^{\prime}\right] z=z^{\prime} \operatorname{dvec}\left\{\left[\Xi(t) \times \kappa_{d}\right] \hat{A}^{\prime}\right\}
$$

and

$$
\left[\zeta(t-u)^{\prime} Z_{u-}\right]=\hat{E}\left[\hat{e} \cdot Z_{t} \mid Z_{u-}\right]
$$


Combining this result with the expression for $\hat{E}\left[\left(\hat{e} \cdot Z_{t}\right)\left(D_{t}-\bar{D}_{t}\right) \mid Z_{0}=z\right]$ completes the proof.

\section{Stochastic discount factor}

An alternative derivation of the continuous-time stochastic discount factor follows from the approach in Duffie and Epstein (1992).

i) Take a monotone transformation of the utility index:

$$
W_{t}=\left(V_{t}\right)^{1-\gamma}
$$

For $\gamma>1$, the case that interests us, this transformation is decreasing, so we will have to make an appropriate sign adjustment.

ii) Notice that $\lambda_{t}$ is the local mean for $W$. Solve (27) for $\lambda_{t}$ :

$$
\begin{aligned}
\lambda_{t} & =\delta(\gamma-1)\left[\log C_{t}+\left(\frac{1}{\gamma-1}\right) \log W_{t}\right] W_{t} \\
& =\Phi\left(C_{t}, W_{t}\right)
\end{aligned}
$$

iii) Following Duffie and Epstein (1992) (see their formula (35)),

$$
\begin{aligned}
S_{t} & =\frac{\Phi_{c}\left(C_{t}, W_{t}\right)}{\Phi_{c}\left(C_{0}, W_{0}\right)} \exp \left[-\int_{0}^{t} \Phi_{w}\left(C_{u}, W_{u}\right) d u\right] \\
& =\left(\frac{C_{0}}{C_{t}}\right)\left(\frac{W_{t}}{W_{0}}\right)^{1-\gamma} \exp \left(-\delta t-\delta(\gamma-1) \int_{0}^{t}\left[\log C_{u}+\left(\frac{1}{\gamma-1}\right) \log W_{u}\right] d u\right) \\
& =\exp (-\delta t)\left(\frac{C_{0}}{C_{t}}\right)\left(\frac{V_{t}}{V_{0}}\right)^{1-\gamma} \exp \left[\delta(\gamma-1) \int_{0}^{t}\left(\log V_{u}-\log C_{u}\right) d u\right] \\
& =\exp (-\delta t)\left(\frac{C_{0}}{C_{t}}\right)\left(\frac{V_{t}}{V_{0}}\right)^{1-\gamma} \exp \left[\delta(\gamma-1) \int_{0}^{t} v \cdot Z_{u} d u\right]
\end{aligned}
$$

where we have placed a minus sign in front $\Phi_{w}$ to offset the fact that we used a monotone decreasing transformation of the utility index. This formula is consistent with our conclusion in Section 6.2. 


\section{References}

Alvarez, Fernando and Urban J. Jermann. 2005. Using Asset Prices to Measure the Persistence of the Marginal Utility of Wealth. Econometrica 73 (6):1977-2016.

Bansal, Ravi and Bruce N. Lehmann. 1997. Growth-Optimal Portfolio Restrictions on Asset Pricing Models. Macroeconomic Dynamics 1:333-354.

Bansal, Ravi and Amir Yaron. 2004. Risks for the Long Run: A Potential Resolution of Asset Pricing Puzzles. The Journal of Finance 59 (4):1481-1509.

Bhamra, Harjoat S., Lars-Alexander Kuehn, and Ilya A. Strebulaev. 2008. The Levered Equity Risk Premium and Credit Spreads: A Unified Framework.

Bonomo, Marco and René Garcia. 1996. Consumption and Equilibrium Asset Pricing: An Empirical Assessment. Journal of Empirical Finance 3 (3):239-265.

Breeden, Douglas T. 1979. An Intertemporal Asset Pricing Model with Stochastic Consumption and Investment Opportunities. Journal of Financial Economics 7 (3):265-296.

Cagetti, Marco, Lars Peter Hansen, Thomas J. Sargent, and Noah Williams. 2002. Robustness and Pricing with Uncertain Growth. The Review of Financial Studies 15 (2):363-404.

Calvet, Laurent E. and Adlai J. Fisher. 2008. Multifrequency Jump-Diffusions: An Equilibrium Approach. Journal of Mathematical Economics 44 (2):207-226.

Campbell, John Y. and John Cochrane. 1999. By Force of Habit: A Consumption-Based Explanation of Aggregate Stock Market Behavior. Journal of Political Economy 107 (2):205251.

Chen, Hui. 2008. Macroeconomic Conditions and the Puzzles of Credit Spreads and Capital Structure. Forthcoming in Journal of Finance.

David, Alexander. 1997. Fluctuating Confidence in Stock Markets: Implications for Returns and Volatility. The Journal of Financial and Quantitatuve Analysis 32 (4):427-462.

Duffie, Darrell and Larry G. Epstein. 1992. Asset Pricing with Stochastic Differential Utility. The Review of Financial Studies 5 (3):411-436.

Epstein, Larry G. and Stanley E. Zin. 1989. Substitution, Risk Aversion, and the Temporal Behavior of Consumption and Asset Returns: A Theoretical Framework. Econometrica 57 (4):937-969. 
Fournié, Eric, Jean-Michel Lasry, Jérôme Lebuchoux, and Pierre-Louis Lions. 1999. Applications of Malliavin Calculus to Monte-Carlo Methods in Finance. Finance and Stochastics $3(4): 391-412$.

Gourieroux, C. and J. Jasiak. 2005. Nonlinear Innovations and Impulse Responses with Application to VaR Sensitivity. Annales d'Economie et de Statistique 78:1-33.

Hansen, Lars Peter. 2007. Beliefs, Doubts and Learning: Valuing Macroeconomic Risk. American Economic Review 97:1-30.

_ 2009. Modeling the Long Run: Valuation in Dynamic Stochastic Economies. FisherSchultz Lecture at the European Meetings of the Econometric Society.

Hansen, Lars Peter and Eric Renault. 2009. Pricing Kernels. Forthcoming in Encyclopedia of Quantitative Finance.

Hansen, Lars Peter and José A. Scheinkman. 2009a. Long-Term Risk: An Operator Approach. Econometrica 77 (1):177-234.

- 2009b. Pricing Growth-Rate Risk. Forthcoming in Finance and Stochastics.

Hansen, Lars Peter, John C. Heaton, Junghoon Lee, and Nikolai Roussanov. 2007. Intertemporal Substitution and Risk Aversion. In Handbook of Econometrics: Volume 6A, chap. 61, 3967-4056. Elsevier.

Hansen, Lars Peter, John C. Heaton, and Nan Li. 2008. Consumption Strikes Back?: Measuring Long-Run Risk. Journal of Political Economy 116:260-302.

Haussmann, Ulrich G. 1979. On the Integral Representation of Functionals of Ito Processes. Stochastics and Stochastics Reports 3 (1):17-27.

Ito, Kiyosi and Shin Chi Watanabe. 1965. Transformation of Markov Processes by Multiplicative Functionals. Annals of Institute Fourier 15:13-30.

Koijen, Ralph S.J., Hanno Lustig, and Stijn van Nieuwerburgh. 2009. The Cross-Section and Time-Series of Stock and Bond Returns.

Koop, Gary, M. Hashem Pesaran, and Simon M. Potter. 1996. Impulse Response Analysis in Nonlinear Multivariate Models. Journal of Econometrics 74 (1):119-147.

Kreps, David M. and Evan L. Porteus. 1978. Temporal Resolution of Uncertainty and Dynamic Choice Theory. Econometrica 46 (1):185-200. 
Lettau, Martin and Jessica Wachter. 2007. Why is Long-Horizon Equity Less Risky? A Duration-Based Explanation of the Value Premium. Journal of Finance 62:55-92.

Lucas, Jr., Robert E. 1978. Asset Prices in an Exchange Economy. Econometrica 46 (6):14291445.

Potter, Simon M. 2000. Nonlinear Impulse Response Functions. Journal of Economic Dynamics and Control 24 (10):1425-1446.

Santos, Tano and Pietro Veronesi. 2008. Habit Formation, the Cross Section of Stock Returns and the Cash-Flow Risk Puzzle. Working paper, University of Chicago.

Wachter, Jessica. 2005. Solving Models with External Habit. Finance Research Letters $2: 210-226$.

Wong, Eugene. 1964. The Construction of a Class of Stationary Markoff Processes. In Stochastic Processes in Mathematical Physics and Engineering, edited by Richard E. Bellman, no. 17 in Proceedings of Symposia in Applied Mathematics, 264-276. American Mathematical Society. 